\title{
Unemployment Insurance with Limited Commitment Wage Contracts and Savings
}

R. Oikonomou

Discussion Paper 2016-6

\section{Institut de Recherches Économiques et Sociales de I'Université catholique de Louvain}




\title{
Unemployment Insurance with Limited Commitment Wage Contracts and Savings *
}

\author{
Rigas Oikonomou ${ }^{\dagger}$ \\ Université Catholique de Louvain
}

February 2016

\begin{abstract}
I present a model of optimal contracts between firms and workers, under limited commitment and with worker savings. In the model, firms provide insurance against unemployment through targeting a frontloaded path of wages which encourages wealth accumulation. I provide analytical results characterising the wage and savings schedules and the path of consumption during employment and unemployment. I then consider how unemployment benefits affect risk sharing through private markets. I find that benefits should be frontloaded; the government has the incentive to drive the allocation to the point where the firm's participation constraint binds. At this point wages are equal to productivity in every period, wealth exceeds the buffer stock level, and consumption and savings drop over time. The drop in the level of consumption during unemployment is mitigated.

Finally, I compare the optimal contract model to the standard heterogeneous agent model whereby wealth is utilized for self-insurance purposes. I show that the two models are equivalent under the optimal UI policy.
\end{abstract}

JEL codes: D52, E21, H31, H53, J41

Keywords: Unemployment Insurance, Incomplete Markets, Optimal Contracts, Limited Commitment, Household Self-Insurance.

*I am grateful to my advisors Rachel Ngai and Chris Pissarides. I also benefited a lot from the comments of Wouter den Haan, Elisa Faraglia, Joachim Jungherr, Gueorgui Kambourov, Jochen Mankart, Albert Marcet, Christian Siegel two anonymous referees and participants at the LSE macro seminar and the Macro Reading group in Autonoma. Special thanks is owed to Athan Zafirov for excellent research assistance. Correspondence Information: Rigas Oikonomou, IRES-CORE, Université Catholique de Louvain, Collège L. H. Dupriez, 3 Place Montesquieu 1348 Louvain la Neuve, Belgium.

${ }^{\dagger}$ Email address: rigas.oikonomou@uclouvain.be 


\section{Introduction}

Whether governments should provide insurance against the risk that individuals face in the labor market has been a long standing debate in economics. Much of the discussion over the scope of public policy centers around the notion that it can crowd out the private insurance arrangements of individuals, with a widespread belief that the scope of policy is limited when such arrangements are in place. Theoretical work seeking to describe this tradeoff is ample: Attanasio and Rios Rull (2000) and Thomas and Worrall (2007) (among others) study models with limited commitment contracts between risk averse households and show that public insurance can crowd out private risk sharing with adverse effects on welfare. Hansen and Imhrohoroglu (1992) and Wang and Williamson (2002) (among others) study the properties of optimal unemployment insurance within the heterogeneous agents model of precautionary savings. They illustrate that unemployment benefits discourage individuals from accumulating wealth, reducing the scope of self insurance.

This paper investigates the effects of unemployment benefits in an economy where private risk sharing is a contract under limited commitment offered by firms to their workforce. The contract is broadly similar to the self enforcing wage models of Thomas and Worrall (1988) and Rudanko $(2009,2011)$ however, in contrast to these papers, I assume that agents have access to a storage technology and can accumulate assets over time. The model is cast in an equilibrium environment whereby individuals choose optimally their savings, and the distribution of wealth is determined endogenously as the outcome of these choices. The model therefore weds the limited commitment contract with the standard framework of heterogeneous agents and wealth accumulation; I depart however from this framework through introducing wages which are set optimally and not assumed equal to labor productivity in every period.

I establish the following results: First, the contract offers a higher wage at the start of the job, to individuals that have low wealth, in order to encourage savings. These savings are used to (partially) insure workers against the risk of unemployment, the only shock to the income process considered in the model. The allocation is stationary after one period, featuring constant consumption, wages, wealth and utility over the life of the match. Second, I show that when agents are initially wealthy, the optimal contract sets wages equal to productivity in every period. In this case, consumption and wealth drop through time until a constant level of assets is reached. This level defines the buffer stock of savings in the model.

As is well known, under limited commitment the optimal allocation needs to satisfy two participation constraints: Both the firm and the worker must be better off in the match than if they separate. In models which feature uncertainty in the level of productivity (e.g. Thomas and Worrall (1988)) this implies that the two parties need to give up risk sharing to satisfy the constraints. In the simple model analyzed in this paper, which assumes that productivity is constant through time, the constraints influence the properties of wealth accumulation and in turn, influence risk sharing against unemployment which depends on assets.

Under the assumptions that I make over preferences I obtain that the only constraint which becomes relevant in equilibrium is the firm's constraint. Indeed, in all the versions of the model considered the allocation corresponds to either an unconstrained (Pareto) optimum or a constrained optimum in which the firm's constraint is binding. The previous results can be interpreted in light of these remarks: An unconstrained Pareto optimal allocation features a constant ratio of marginal 
utilities over time. When a participation constraint is hit the level of consumption of the party which needs to be made better off increases (see Kocherlakota (1996)). Since the firm is assumed risk neutral, the ratio of marginal utilities coincides with the marginal utility of the worker. When the participation constraint of the firm is binding the worker's consumption drops, and when it is not binding, her consumption remains constant. The model yields the sharp prediction that in the first case, assets exceed the buffer stock and in the second, they fall short of the buffer stock. These properties are established analytically.

I then turn to investigate the effects of government provided unemployment insurance with particular focus on the timing of benefits. The key finding is that the government should frontload unemployment insurance; it is preferable (welfare maximizing) to pay a large benefit in the first period (quarter) of the unemployment spell and set benefits to a low but positive level in subsequent periods.

To understand this result it is important to outline the following: First, as is common in the optimal contracts literature, I assume that the firm and the worker discount the future at the same rate, $\beta$. Second, I assume that assets earn a gross return $r$, but under incomplete financial markets it must be that $\beta r<1$ for the equilibrium to be well defined (see Huggett (1993)). With these assumptions the firm has access to a storage technology which earns a superior rate of return than assets do in the market. When it is optimal for the worker to accumulate wealth, she must borrow from the firm (through a frontloaded wage) and increase her endowment. The cost of borrowing is proportional to the difference between the two rates of return, and because borrowing is costly, complete insurance against unemployment becomes suboptimal. The only case where consumption does not drop considerably in unemployment is when the firm's participation constraint is binding. In this case, the wealth level of the agent already exceeds the buffer stock and she does not have bear the cost of borrowing from the firm.

Consider now a UI scheme which pays high benefits in the first period of unemployment and subsequently reduces unemployment income considerably. Given this policy, the newly unemployed agent is induced to save to ward off the risk of a prolonged spell. Since in the model (and in the data) a large fraction unemployed individuals find jobs after one quarter, they arrive to their new jobs with a high wealth endowment. Their wealth exceeds the buffer stock and the participation constraints bind in equilibrium. For this reason, UI policies which concentrate payments in the first period of unemployment perform considerably better. The importance of this channel is shown to depend on the level of interest rates.

This paper is related to several strands in the literature. First, there is a considerable literature of models of heterogenous agents and precautionary savings. As is well known, these models predict that i) individuals accumulate savings (and consumption increases over time) until the buffer stock is built and ii) if the level of wealth exceeds the buffer stock, then consumption and wealth drop through time (see for example Aiyagari (1994) among many others). A key innovation of my paper is to replace the assumption that workers are paid the marginal product each period with an optimal contracting scheme which is widely viewed as an alternative to wage models without commitment. The intertemporal behavior predicted by the model is similar, however, as I explained previously, wealth accumulation takes place in only one period and consumption remains constant when the agent wants to save. When the firm's participation constraint is binding the two models become 
identical.

Second, limited commitment models with savings are not abundant in the literature. An important contribution is that of Ligon, Thomas and Worrall (2000, hereafter LTW), the first who studied this problem in the context of mutual insurance between risk averse households. ${ }^{1}$ They illustrate that intertemporal optimization leads to an Euler equation featuring terms which capture the effect of wealth on the limited commitment friction. In my model because the firm is risk neutral and the firm's constraint is the only one which is relevant in equilibrium, the standard Euler equation holds as an equality. The predictions of the theory for wealth and consumption are, therefore, not driven by any distortion associated with limited commitment; individuals optimize intertemporally as they do in standard theories of heterogeneous agents.

These issues have been recently studied in Karaivanov and Martin (2015, KM) who characterize the properties of optimal consumption and savings in the context of equilibria without commitment where wealth is the sole state variable (Markov perfect contracts). Though their analysis includes a comparison between the Markov contract and the limited commitment allocation, they focus on the case where the firm can commit, the worker is indifferent (initially) between remaining in and opting out of the contract. As I previously explained, in my model the firm's participation constraint becomes the crucial constraint in equilibrium, the focus is on the case where the worker extracts all the surplus from the match initially. Moreover, whereas KM formalize income risks through a stochastic endowment process, I model them as unemployment risks. My results are complementary to theirs.

The prediction of the theory I present, that wages and wealth are linked, is not uncommon in the literature. Models which determine wages through Nash bargaining and allow workers to save (for example see Krusell et al (2010) and Nakajima (2012)), reach a similar presumption, even though the wage profiles predicted by these theories are quite different from the ones I obtain in my model. To show that the frontloaded wage property is not at odds with the data, I argue that it can be interpreted as a constant wage and severance payment scheme. I then utilize the model to evaluate UI policies in the United States. In some states benefits are reduced when individuals receive severance compensation, in others it is not the case. The second group of states is closer to the welfare maximizing policy predicted by the model. Pissarides (2010) is another paper which explores the interplay between severance payments and unemployment benefits.

Finally, as discussed previously, the paper is related to the sizable literature which considers the impact of unemployment insurance within the heterogeneous agent framework. Hansen and Imhrohoroglu (1992) are concerned with characterising the optimal (permanent) level of benefits when individuals receive job opportunities exogenously but may choose to reject them. Wang and Williamson (2002) consider an environment where unemployed and employed individuals exert effort, the former to find jobs and the latter to keep their jobs. They find that the welfare gains from unemployment insurance are not substantial. The model presented in this paper is closer to Wang and Williamson (2002) but it leaves out 'job retention' effort. I focus on an environment where individuals lose their jobs through the arrival of an exogenous 'destruction' shock, this simplification is made for the sake of highlighting the properties of the optimal contract.

The paper proceeds as follows: Section 2 presents the economic environment. Section 3 discusses

\footnotetext{
${ }^{1} \mathrm{~A}$ more recent contribution is Voena (2015) who models the savings decisions of married couples under limited commitment.
} 
the implications of the optimal contract. Section 4 calibrates the model. Section 5 contains the main results on the effects of unemployment insurance. Section 6 presents several robustness exercises and extensions. A final section concludes.

\section{The Model}

\subsection{Economic Environment}

Preferences. There is a continuum (measure one) of infinitely lived, risk averse agents with preferences of the following form:

$$
E_{0} \sum_{t=0}^{\infty} \beta^{t}\left(\log \left(c_{t}\right)-v\left(s_{t}\right)\right)
$$

$c_{t}$ denotes the consumption of a general multipurpose good. $s_{t}$ is a search intensity variable with $s_{t} \in[0,1]$ and $v\left(s_{t}\right)$ denotes the disutility of search. $v$ satisfies $v(0)=0, v_{s}\left(s_{t}\right)>0$ and $v_{s s}\left(s_{t}\right)>0$. $\beta$ is the discount factor. As in Wang and Williamson (2002) and Young (2004) a model period $t$ represents one quarter.

Employment Opportunities. Every period, a fraction $e=1-u$ of all individuals are employed, matched with firms, the remaining $u$ agents are unemployed, waiting for a job offer to arrive. The arrival rate of offers to unemployed individuals is given by $\gamma\left(s_{t}\right)$, where $\gamma$ is a technology mapping search effort to a job finding probability. When matched, the employed agent (worker) produces $y$ units of output per period. The firm does not search actively for a worker and when the job starts it is assumed to earn zero profits in expectation. Moreover, employed agents also don't search (hence $s_{t}=0$ for them), their matches terminate at an exogenous rate $\lambda$ per unit of time. When this happens they become unemployed.

Unemployment Insurance. Let $j=0,1,2, \ldots$ denote the number of periods that an individual has spent in unemployment prior to the current period. An agent with an index $j$ is at her $j+1$ period. $j=0$ applies to a newly unemployed agent. The government provides insurance against unemployment in the form of benefits denoted by $b_{j}$. Benefits depend on the index $j$ to show that the income received varies with the duration of the spell. Not all unemployed individuals are eligible for benefits: There is a horizon $m$ beyond which unemployment income is equal to zero. Therefore, $b_{j}=0, \quad j \geq m$ and $b_{j}>0, j<m$. For the UI scheme in the US we have that $m=2$.

To finance benefits, taxes $(\tau)$ are levied on employed individuals each period. It is assumed that the government runs a balanced budget. Therefore, we have that $e \tau=\sum_{j<m} u_{j} b_{j}$, where $u_{j}$ denotes the number of unemployed agents who are running their $j+1$ period of joblessness.

Financial Markets. Financial markets are incomplete. Agents can hold a non-state contingent asset, subject to an ad hoc (no borrowing) constraint. The gross interest rate on the asset is denoted by $r$. Following Wang and Williamson (2002) I consider an economy without capital. I impose $\beta r<1$ so that asset positions do not diverge (see Huggett (1993) and Aiyagari (1994)). 


\section{$2.2 \quad$ Value Functions}

Employed Agents. As discussed previously, each match generates a per period output equal to $y$. Because firms earn zero profits at the start of the match, one possible firm-worker contract is to pay a wage $y$ each period and let the worker accumulate savings to self-insure against the job separation risk. This arrangement is considered by most papers in the literature. However, it is not (necessarily) optimal; insofar as the firm is risk neutral and the worker is risk averse there could be a different payment profile that (Pareto) dominates the flat wage contract.

I describe the Pareto optimal allocation in this subsection. To do so I utilize the recursive formulation of LTW which is broadly applied in the literature of self-enforcing labor contracts. Let $J_{t}$ be the present discounted (profit) value of the firm, $a_{t}$ the stock of wealth of the worker, and $W\left(a_{t}, J_{t}\right)$ the lifetime utility of the worker. The optimal contract maximizes $W$ subject to a sequence of constraints. Among these are the participation constraints $J_{t} \geq 0$, and $W_{t} \geq U\left(a_{t}, 0\right)$ where $U\left(a_{t}, 0\right)$ is the lifetime utility of a newly unemployed individual. These constraints ensure that the optimal allocation makes both the firm and the worker (weakly) prefer to be matched rather than to separate for all $t$.

The recursive representation of the contract is: ${ }^{2}$

$$
\begin{gathered}
W(a, J)=\max _{a^{\prime} \geq 0, J^{\prime}, w} \log (c)+\beta\left[\lambda U\left(a^{\prime}, 0\right)+(1-\lambda) W\left(a^{\prime}, J^{\prime}\right)\right] \\
\text { subject to: } a^{\prime}=r a+w-\tau-c \\
J=y-w+\frac{1-\lambda}{R} J^{\prime} \\
J^{\prime} \geq 0 \quad \text { and } \quad W\left(a^{\prime}, J^{\prime}\right) \geq U\left(a^{\prime}, 0\right)
\end{gathered}
$$

(3) is the worker's budget constraint. (4) is the so called promise keeping constraint; it imposes that the firm's expected profits equal $J$. The firm earns $y-w$ this period, where $w$ denotes the wage, and discounts the (expected) future profit value $\left((1-\lambda) J^{\prime}\right)$ at rate $\frac{1}{R}$. The worker chooses $a^{\prime}, w$ and the continuation utility $J^{\prime}$ for the firm.

Two remarks are in order: First, note that it is common in the literature to represent optimal contracts letting the firm choose allocations subject to the worker receiving a predetermined level of utility. This is the benchmark followed by Thomas and Worrall (1988), Rudanko (2009), KM and many others. I chose to work with $W(a, J)$ for convenience; it allows me to (subsequently) define in the unemployed worker's program, the payoff from finding a job using the solution to (2) and setting $J=0$. This is without loss of generality. One can derive the same optimal allocation from (2) as from a Bellman equation which maximizes firm profits. ${ }^{3}$

Second, even though it holds that $J=0$ at the start of each match, it is important to consider values for $J$ different from zero in the value function. As will become evident later, for a wage profile that is not constant over time we have that $J_{0}=0$ but $J_{t} \neq 0$ for $t>0$; the total wage paid between periods 0 and $t-1$ is not equal to $t y$. In a subsequent paragraph I describe how the sequence of $J_{\mathrm{S}}$ evolves over time.

Unemployed Agents. Equilibrium payoffs for unemployed individuals solve the following func-

\footnotetext{
${ }^{2}$ I drop all time subscripts from the Bellman equation and use primes to denote next period variables.

${ }^{3}$ Assets are contractible and observable to the firm. When the firm maximizes profits, it chooses wages, assets and the promised utility to the worker as in KM. See derivations in the online appendix.
} 
tional equation:

$$
\begin{gathered}
\left.U(a, j)=\max _{a^{\prime} \geq 0, s} \log (c)-v(s)+\beta \gamma(s) W\left(a^{\prime}, 0\right)+\beta(1-\gamma(s)) U\left(a^{\prime}, j+1\right)\right) \\
\text { subject to: } a^{\prime}=r a+b_{j}-c
\end{gathered}
$$

From program (6) we can derive the following optimality conditions:

$$
\begin{gathered}
U_{a}\left(a_{t}, j\right)=\frac{r}{c_{t}^{u}} \quad \text { (envelope) } \\
\left.-v_{s}\left(s_{t}\right)+\beta \gamma_{s}\left(s_{t}\right)\left(W\left(a_{t+1}, 0\right)-U\left(a_{t+1}, j+1\right)\right) \leq 0 \quad \text { (with equality if } s_{t}>0\right) \\
\frac{1}{c_{t}^{u}} \geq \beta r\left(\frac{1-\gamma\left(s_{t}\right)}{c_{t+1}^{u}}+\frac{\gamma\left(s_{t}\right)}{c_{t+1}^{e}}\right) \quad\left(\text { with equality if } a_{t+1}>0\right)
\end{gathered}
$$

${ }^{4}$ where $c_{t}^{u}\left(c_{t}^{e}\right)$ denotes the agent's consumption in unemployment (employment) in $t$. The definition of the competitive equilibrium is given in the appendix.

\section{Implications}

This section studies the implications of the optimal contract. I derive theoretically the optimal wage, consumption and asset paths. To conserve space I show the paths in the case where the worker's participation constraint never binds, the only constraint that may bind is the firm's constraint. As discussed previously, this property characterizes all of the models solved in this paper. It is a consequence of the assumptions I make over the relative discount rates of the worker and the firm (see discussion and examples in the online appendix). ${ }^{5}$ My results derive under the following additional assumptions:

Assumption A.1. The discount factor of the firm satisfies $\frac{1}{R}=\beta$. Moreover, it holds that $r<R=\frac{1}{\beta}$ and $r \geq 1$.

Assumption A.2. i) It holds that $W(a, 0)>U(a, j)$ for all $j$. ii) $U(a, j)$ is strictly increasing in a and strictly concave.

A.1 imposes that the firm and the worker discount the future at equal rates; this is standard in the literature of self enforcing labor contracts. A.2 lists desirable technical properties of the value functions. i) requires that the surplus from a new match is strictly positive and at all wealth levels. Since I have assumed that the worker does not derive disutility from working, i) requires that unemployment benefits are not that high so that agents set $s(a, j)=0$ for some $j$. Under all the benefit schemes considered subsequently A.2 will hold.

\footnotetext{
${ }^{4}$ Clearly when $b_{j}=0$ the constraint $a_{t+1} \geq 0$ will not bind since it coincides with the natural borrowing limit of the unemployed agent (see Aiyagari (1994)). In this case the Euler equation holds as an equality.

${ }^{5}$ Leaving out the worker's constraint is (technically) equivalent to solving the optimal contract under one sided limited commitment (e.g. Rudanko (2009)). In other words, I could setup the Bellman equation (2) ignoring the constraint $W\left(a^{\prime}, J^{\prime}\right) \geq U\left(a^{\prime}, 0\right)$. The more complete formulation which includes the constraint serves to highlight that the condition $W\left(a^{\prime}, J^{\prime}\right)>U\left(a^{\prime}, 0\right)$ is a result of the worker's optimization, not an ad hoc assumption. Though I cannot provide an analytical proof for this property, I provide an intuition based on the features of the optimal allocation identified in this section. I leave it to the online appendix to rederive the optimality conditions including the terms which reveal the influence of the worker's constraint.
} 


\subsection{Intertemporal Behavior}

From the first order conditions of program (2) (see online appendix) we can derive the following equations:

$$
\begin{gathered}
\frac{1}{c_{t}^{e}} \beta(1-\lambda)=\frac{1}{c_{t+1}^{e}} \beta(1-\lambda)-\phi_{t} \\
\frac{c_{t+1}^{e}}{c_{t+1}^{u}}=1+\frac{\left(\frac{1}{\beta}-r\right)}{r \lambda}-\frac{\chi_{t} c_{t+1}^{e}}{r \lambda \beta}-\frac{1}{\beta(1-\lambda)} \frac{1}{r \lambda \beta} \phi_{t} c_{t+1}^{e}
\end{gathered}
$$

where $\phi_{t}$ is the multiplier on the participation constraint of the firm, $\chi_{t}$ is the multiplier on the borrowing constraint.

Consumption in employment. (7) gives the optimal consumption path. If $\phi_{t}=0$ we have $c_{t}^{e}=c_{t+1}^{e}$; consumption remains constant over the life of the match. However, when $\phi_{t}>0$ (the firm's participation constraint binds) we get $\frac{1}{c_{t}^{e}}<\frac{1}{c_{t+1}^{e}} \rightarrow c_{t}^{e}>c_{t+1}^{e}$; the level of consumption drops over time.

As discussed previously, the marginal utility $\frac{1}{c_{t}^{e}}$ represents the relative weight of the firm and the worker in the Pareto optimal allocation. ${ }^{6}$ If the participation constraint is slack the weight remains constant over time. If the constraint binds in $t+1$, then consumption must drop to ensure that the relative weight of the firm increases.

Consumption in unemployment. (8) gives the consumption of the worker if she remains employed in $t+1$ relative to her consumption if she becomes unemployed. The ratio $\frac{c_{t+1}^{e}}{c_{t+1}^{u}}$ determines the scope of insurance of the optimal contract; if consumption drops considerably in unemployment the insurance value is smaller and conversely, if consumption does not drop then the contract can completely insure the worker against an unemployment spell which lasts for one period. Insurance is not perfect even when $\frac{c_{t+1}^{e}}{c_{t+1}^{u}}=1$ because unemployment typically lasts for more than a period and assets and consumption drop during unemployment spells. When the match is destroyed the worker is beyond the reach of the firm and must rely on government benefits to mitigate further consumption loses.

Off corners the right hand side of (8) becomes: $1+\left(\frac{1}{\beta r}-1\right) \frac{1}{\lambda}$. The unemployment spell leads to a drop in consumption that is proportional to the ratio of $\frac{1}{\beta}$ over $r$. The worker can insure her consumption through increasing the wealth level $a_{t}$. As I will later show, to do this 'optimally' she will 'borrow' from the firm, where 'borrowing' means to receive a higher wage today and invest in $a_{t}$. From the promise keeping constraint, the interest cost of this 'loan' is $R \equiv \frac{1}{\beta}$; Since $\frac{1}{\beta}>r$ borrowing from the firm is costly. For this reason $\frac{c_{t+1}^{e}}{c_{t+1}^{u}}$ grows with the difference between $\frac{1}{\beta}$ and $r .^{7}$

Euler equation. The Euler equation of the model is given by:

$$
\frac{1}{c_{t}^{e}}=\beta r\left[(1-\lambda) \frac{1}{c_{t+1}^{e}}+\lambda \frac{1}{c_{t+1}^{u}}\right]+\chi_{t}
$$

\footnotetext{
${ }^{6}$ In the online appendix I utilize a Lagrangian formulation to show this. This interpretation is standard in the literature (see LTW and Marcet and Marimon (2002)).

${ }^{7}$ Another way of saying this is that when employed, the agent effectively has access to two types of assets: a non-state contingent asset with return $r$ (subject to the borrowing restriction) and a state contingent one which pays out a return $\frac{1}{\beta}>r$ in the event she remains employed (see for example Pissarides (2010)). To insure consumption the worker must short the first asset and take a long position in the second.
} 
The standard equality between the cost of an extra unit of savings today $\left(\frac{1}{c_{t}^{e}}\right)$ and the future benefit $\left(\beta r\left[(1-\lambda) \frac{1}{c_{t+1}^{e}}+\lambda \frac{1}{c_{t+1}^{u}}\right]\right)$ holds in the model if $a_{t+1}>0$. As discussed previously, this property derives from the fact that the firm's constraint is the only one that may bind in equilibrium. ${ }^{8}$

\subsection{Wage Profiles}

Unconstrained Optimum. I now characterize the behavior of wages in the model. I first characterize the optimal wage path in the case of an unconstrained optimum, subsequently, I explain the properties of the wage profile when the firm's constraint is binding.

Proposition 1. Assume that $\chi_{t}=0$ and $\phi_{t}=0$ for $t=0,1,2, \ldots$. We can show that: i) Wages are constant for $t=1,2, \ldots$ i.e. $w_{t}=\bar{w}, t>0$. ii) The initial wage could either satisfy $w_{0}>\bar{w}$ or $w_{0}=\bar{w}$. In the latter case it must be that $\bar{w}=y$.

Proof. i) follows from the fact that $c_{t}^{e}=c_{t+1}^{e}$ (e.g. (7)). Since the ratio of the marginal utility of consumption in employment and unemployment is constant and $\frac{c_{t+1}^{e}}{c_{t+1}^{u}}=1+\frac{\left(\frac{1}{\beta}-r\right)}{r \lambda}$ (from (8)), by the envelope condition on the unemployed agent's value function $\left(U_{a^{\prime}}\left(a_{t+1}, 0\right)=\frac{r}{c_{t+1}^{u}}\right)$ and the fact that $c_{t+1}^{u}$ is constant for $t \geq 0$, we have that $a_{1}=a_{2}=a_{3}=\ldots$. From the worker's budget constraint we have: $w_{t}=-a_{t+1}+r a_{t}-\tau-c_{t}^{e}$. Since the RHS of this equation is constant we can write $w_{t}=\bar{w}$ for $t>0$.

ii) states that the initial wage could be larger than $\bar{w}$. By the stationarity of wages from $t=1$ onwards and the firm's promise keeping constraint we can write: $J_{0}=0=y-w_{0}+\frac{y-\bar{w}}{1-\beta(1-\lambda)} \cdot{ }^{9}$ It follows that if $w_{0}>\bar{w}$ then $w_{0}>y$ and $\bar{w}<y$ (otherwise $J_{0} \neq 0$ ).

Could the initial wage be smaller than $y$ thus leading to an extraction of the worker's wealth endowment? The answer is no. In this case the wage profile violates the firm's participation constraint. Assume the converse: Let the firm pay $w_{0}<y$ in the initial period. Under zero initial profits the firm must then offer $\bar{w}>y$. It earns a negative profits from $t=1$ onwards. This violates participation.

Proposition 1 tells us that it may be optimal for the worker to frontload wage payments. As discussed in the previous subsection, the worker may find it optimal to 'borrow from the firm' and invest in $a_{t}$, this allows her to partially insure against the unemployment risk. Borrowing from the firm occurs through the frontloaded wage. If it is optimal to set $w_{0}>y$ we have that $a_{t}>a_{0}$, $t \geq 1^{10}$

Notice that Proposition 1 imposes $\phi_{t}=0$ for all $t$. This may seem a very restrictive case; if we have $\phi_{t}>0$ for some $t>0$ then the wage profile is not the one described previously. The following result establishes that if the firm's constraint does not bind initially it will never bind, the wage profile is the one described in Proposition 1.

8 KM obtain, in their no-commitment model, the standard Euler equation when firms earn zero profits. Here this property does not hinge on the zero profit condition; the Euler equation holds at $J>0$ insofar as the worker's participation constraint does not bind. See online appendix for a version of the Euler equation in which the worker's constraint may bind (as in LTW).

${ }^{9}$ The firm's expected profits satisfy: $J_{t}=\frac{y-\bar{w}}{1-\beta(1-\lambda)}$ for $t=1,2, \ldots$ since wages are constant.

${ }^{10}$ In the unconstrained optimum we have that: $c_{t}^{e}=(r-1) a_{1}+\bar{w}-\tau$ for $t=0,1,2, \ldots$. The initial budget constraint of the worker gives: $a_{1}=r a_{0}+w_{0}-\tau-c_{0}^{e}=r a_{0}+w_{0}-\tau-\left[(r-1) a_{1}+\bar{w}-\tau\right]$. Therefore, $a_{1}-a_{0}=\frac{1}{r}\left(w_{0}-\bar{w}\right)$. A higher $w_{0}$ leads to a larger stock of wealth. 
Proposition 2. Assume that $\chi_{t}=0, t=0,1,2, \ldots$ and $\phi_{0}=0$. We can show that $\phi_{t}=0$ for $t=1,2, \ldots$.

For the sake of brevity the proof is provided in the appendix.

From Proposition 2 we can easily show that if the firm's constraint doesn't bind in a given period $t$ it will not bind in $t+1, t+2, \ldots$. This property follows from the recursive program and will prove useful subsequently.

Now consider the profile of firm profits implied by Propositions 1 and 2 . We have that $J_{0}=0$ for all matches, however, $J_{t}=\bar{J}>0, t \geq 1$ (where $\bar{J}$ is constant) when $w_{0}>y$. This explains why it is important to consider values of $J$ exceeding zero in the Bellman equation.

The firm's participation constraint. Let me now derive the shape of the wage profile under the assumption that the firm's constraint binds. The following property is useful:

Proposition 3. Suppose that $\phi_{T}>0$ for some $T>0$. Then it must be that $\phi_{t}>0$ for $t=0,1,2, \ldots, T-1$. In words, if the participation constraint of the firm binds in $T$ then it binds in all periods preceding $T$.

Proof. Suppose the converse: assume that the constraint binds in $T$ but does not bind in some $t<T$. This is in contradiction with Proposition 2 (and the property that if the constraint doesn't bind in one period, it never binds again). Moreover, as we previously saw, the allocation is stationary when the participation constraint does not bind. Assets, wages and consumption are constant over time. It follows that the payoffs for the worker and the firm $\left(W\left(a_{t}, J_{t}\right)\right.$ and $\left.J_{t}\right)$ are also constant. If $J_{t}>0$ for $t=1,2, \ldots T$ then $J_{T+1}>0$ and therefore $\phi_{T}=0$.

Proposition 4. Suppose that $\phi_{t}>0$ for $t=0,1,2, \ldots T$. Then, wages are equal to $y$ for all $t$.

Proof. See appendix.

\subsection{Buffer Stock of Savings}

We have seen that the optimal contract gives to the worker a frontloaded wage which allows her to build a stock of assets and insure against unemployment. After the initial investment the allocation is stationary and assets remain constant through time. This holds insofar as the firm's participation constraint does not bind. If it does bind, then consumption drops through time, wages are constant and equal to $y$ and assets are therefore reduced.

In this subsection I highlight an additional feature of the model. I show that the state space can be divided into two regions: when $a_{0}$ is low, wages are frontloaded and assets increase between periods 0 and 1; when $a_{0}$ is large then the firm's constraint is binding. This implies that the optimal contract features a buffer stock of savings. This property is common with the standard model of heterogeneous agents. I explain here the differences between the two frameworks.

To show the above, I use a setup which I can solve analytically. Unfortunately, it is not possible to prove the buffer stock property more generally; however, it holds in all the simulations of the model considered in this paper. ${ }^{11}$ The following assumptions are employed in this section:

\footnotetext{
${ }^{11}$ In the heterogeneous agents literature the buffer stock of savings behavior is also not shown analytically. One can establish that savings diverge when $\beta r \geq 1$, however, there is no analytic proof showing that savings converge
} 
Assumptions A.3: i) unemployment is an absorbing state; employed individuals face a constant probability $\lambda$ of getting fired; when the separation shock arrives agents remain unemployed forever. ii) benefits equal zero at all horizons and therefore taxes are also zero. iii) to further simplify, I assume that $r=1$.

For brevity I leave all of the derivations to the online appendix. I obtain the following:

Result 1. Under A.3, the optimal wage satisfies: $\bar{w}=a_{0}(1-\beta)+\frac{y(1-\beta)}{1-\beta+\beta \lambda} \leq y$ when $a_{0}(1-\beta) \leq$ $y \frac{\beta \lambda}{1+\beta \lambda-\beta} \equiv \widetilde{a}$ (unconstrained optimum). However, if $a_{0}(1-\beta)>\widetilde{a}$ we have $w_{0}=\bar{w}=y$ (the firm's constraint binds).

$\frac{\widetilde{a}}{(1-\beta)}$ is the buffer stock of savings in the model. If $a_{0}>\frac{\widetilde{a}}{(1-\beta)}$ then $c_{t}^{e}>y$ and assets are reduced over time. When $a_{0}=\frac{\widetilde{a}}{(1-\beta)}$ we have $c_{t}^{e}=y$ forever.

Result 2. Let $g^{O C}\left(a_{0}, 0\right)$ represent the optimal savings schedule in the first period of the optimal contract. We have that:

$$
g^{O C}\left(a_{0}, 0\right) \begin{cases}=a_{0}+\frac{1-\beta}{1-\beta+\beta \lambda}\left[\frac{\widetilde{a}}{1-\beta}-a_{0}\right] & \text { if } a_{0} \leq \frac{\widetilde{a}}{1-\beta} \\ <a_{0} & \text { if } a_{0}>\frac{\widetilde{a}}{1-\beta} .\end{cases}
$$

where the expression for $g^{O C}\left(a_{0}, 0\right)$ in the unconstrained optimum follows from Result 1 . To characterize $g^{O C}\left(a_{0}, 0\right)$ in the case where assets exceed the buffer stock the following result is useful.

Result 3. Suppose that $a_{0} \geq \frac{\widetilde{a}}{(1-\beta)}$. Then $W(a, 0)=\widetilde{W}(a)$ where $\widetilde{W}(a)$ solves the following Bellman equation:

$$
\widetilde{W}(a)=\max _{a^{\prime} \geq 0} \log \left(-a^{\prime}+a+y\right)+\beta\left[\lambda U\left(a^{\prime}, 0\right)+(1-\lambda) \widetilde{W}\left(a^{\prime}\right)\right]
$$

In other words, when the firm's constraint binds, the worker solves the Bellman equation of the flat wage contract, the standard model of heterogeneous agents and wealth accumulation. This clearly follows from Proposition 4; since wages always equal $y$, we have that $J_{t}=0 \forall t$. Effectively, $J$ can be dropped from the list of state variables in the optimal program. $\widetilde{W}(a)$ makes use of this property.

From Result 3 it follows readily that $g^{O C}\left(a_{0}, 0\right)=g^{H A}\left(a_{0}\right)$ (where $g^{H A}$ is optimal savings in the heterogeneous agent model) whenever $a_{0}>\frac{\widetilde{a}}{(1-\beta)} \cdot{ }^{12}$ The difference in the savings behavior between the two models derives from when $a_{0}$ falls short of the buffer stock. It is well known, that in the heterogeneous agents framework precautionary savings gradually increase over time until the buffer stock is built (e.g. Deaton (1991) and Aiyagari (1994)). The worker's consumption also increases until it reaches the stationary point $y$. The optimal contract model, however, predicts that when $a_{0}<\frac{\widetilde{a}}{(1-\beta)}, c_{t}^{e}$ is constant, and from $t=1$ onwards assets will also remain constant. Hence, the wealth level $\frac{\widetilde{a}}{(1-\beta)}$ is practically never reached unless the worker starts with this initial level.

Figure 1 shows the savings schedules. The solid line represents $g^{O C}\left(a_{0}, 0\right)$, the dashed line $g^{H A}\left(a_{0}\right)$. The dashed-dotted line is at 45 degrees. The shaded area represents the range of wealth over which

under $\beta r<1$ (see for example Ljungqvist and Sargent (2000)). The buffer stock is typically demonstrated graphically, the graphs summarize the numerical solution to the models. I could have followed this approach but the analytical version of the model employed in this subsection is useful to demonstrate the wage profile derived in the previous Propositions.

${ }^{12}$ It is straightforward to show that the the buffer stock under heterogeneous agents is also equal to $\frac{\widetilde{a}}{(1-\beta)}$. 
the firm's constraint binds and wealth exceeds the buffer stock. The two schedules coincide within the shaded area, however, outside the shaded region (unconstrained optimum) the optimal contract features higher savings for any $a_{0}$.

These differences in savings have important effects on the scope of insurance through assets in the two models. From Figure 1 we see that if workers have low wealth, they build a large wealth endowment much faster under the optimal contract. In contrast, in the flat contract, assets will slowly accumulate, but insofar as the worker remains employed for many periods, they will converge to $\frac{\widetilde{a}}{(1-\beta)}$. The optimal contract can better insure workers against the risk of an early dismissal, under the flat contract the worker may ultimately possess a larger wealth stock.

\subsection{Borrowing Constraints and Wages}

So far my derivations left out the borrowing constraint. It was assumed that $\chi_{t}=0$ for all $t$. This omission was deliberately made; in principle, this constraint will not bind, the reason being that insofar as the worker experiences a drop in income during unemployment, she will always hold positive assets. ${ }^{13}$ This however, will not hold in all of the benefit schemes considered in this paper. As I will later show, when the government provides a very large benefit in the first period of the unemployment spell, then it maybe optimal to set $a_{t}=0$ for some $t$, not to invest in assets. To deal with this case, in this subsection, I briefly explain what happens when $\chi_{t}>0$. The following Proposition summarizes the main takeaway:

Proposition 5. Suppose that the optimal contract sets $\chi_{t}>0$ for some $t>0$. Then the firm's participation constraint binds in every period.

Proof. Let us first assume that the optimum is unconstrained. Since assets are constant after $t=1$ we have that $\chi_{0}=\chi_{1}=\ldots>0$ (the borrowing constraint always binds). Let the worker initially have assets $a_{0} \geq 0$. Then, from the budget constraints we have $c^{e}=w_{0}-\tau+\left(r a_{0}-a_{1}\right)=w_{0}-\tau+r a_{0}$ and also $c^{e}=\bar{w}-\tau$. Therefore, $w_{0}=\bar{w}-r a_{0}$.

Consider first, the case where $a_{0}=0$. We have that $w_{0}=\bar{w}=y$. Second, assume that $a_{0}>0$; it holds that $w_{0}<\bar{w}$. Hence, $w_{0}<y$ and $\bar{w}>y$. This violates the firm's participation constraint. It follows readily that $\phi_{0}>0$. From Proposition 4 we know that wages equal $y$ in all periods (hence $\left.\phi_{t}>0, t=1,2, \ldots\right)$.

Second, assume that $\chi_{t}>0$ for $t>0$ and $a_{0}, a_{1}, \ldots, a_{t-1}>0=a_{t}$. In this case the worker's assets are decreasing over time (or at least they are decreasing between $t-1$ and $t$ ). From the previous results we know that this can only be when the participation constraint of the firm is binding. From Proposition 3 we know that if $\phi_{t}>0$ we must have $\phi_{0}, \phi_{1}, \ldots, \phi_{t-1}>0$. The firm's constraint binds in all periods before $t$. From Proposition 4 we know that wages are equal to $y$ in all periods and therefore also $\phi_{t+1}, \phi_{t+2}, \ldots>0$.

\footnotetext{
${ }^{13}$ This is not a tight argument. The worker may borrow an amount slightly lower than $\frac{b_{0}}{r^{2}}+\frac{b_{1}}{r}$ and pay back the loan almost surely.
} 


\section{Calibration}

Following Wang and Williamson (2002) I assume that the search function is of the following form: $\gamma(s)=1-e^{(-\bar{\gamma} s)}$ where $\bar{\gamma}$ is a constant. The cost of search is given by $v(s)=s^{\delta}$. I set $\delta=2$.

To pin down the separation rate $\lambda$ I assume, that over a monthly horizon workers face a $2.5 \%$ probability of losing their job. This value is well within the range considered in the search and matching literature. Since one period in the model corresponds to one quarter, I have to recover from this assumption the quarterly value for $\lambda$. To accomplish this I first assume that the stationary unemployment rate is $6.2 \%$ (average in the CPS over the years 1994-2011). This gives me a value for the monthly job finding probability of 0.3782 (denote this by $\gamma^{\text {monthly }}$ ). Then, the number of unemployed individuals that have a duration of up to one quarter is given by $\bar{u}_{0} \equiv(1-u) \lambda^{\text {monthly }}\left(1+1-\gamma^{\text {monthly }}+\left(1-\gamma^{\text {monthly }}\right)^{2}\right)$. The quarterly value for $\lambda$ is equal to $\frac{\bar{u}_{0}}{1-u}$ since the stationary unemployment rate applies at all horizons. Hence, $\lambda=0.0503 . \bar{\gamma}=2.54$ is chosen to hit the unemployment rate target.

The baseline benefit scheme is calibrated to the current UI scheme in the US. Individuals receive $50 \%$ of their past income for the first two quarters in unemployment and subsequently receive zero benefits. ${ }^{14}$ To simplify, I set $b_{j}=0.5 y$ for $j=0,1$ and $b_{j}=0$ for $j \geq 2$. This is roughly consistent with the above, since individuals in the model on average earn $y$ every period. I normalize the value of $y$ to unity.

Finally, I calibrate the discount rate and the market interest rate as follows: I choose a value for $r$ equal to one as Wang and Williamson (2002) do. This means that workers have access to a storage technology and they earn zero return on their savings. For parameter $\beta$ which given $r$ governs consumption loses in unemployment, I target a value so that the model yields an average (over an annual horizon) consumption loss suffered by agents that experience unemployment of 6.8\%. This is consistent with the empirical evidence presented in Gruber (1997). This procedure gives $\beta=0.990062$.

\section{The Impact of Unemployment Benefits}

\subsection{Wages, Savings and Consumption under the Baseline UI scheme}

Figure 2 shows the wage schedule offered in the first period of the match (solid-blue line), as a function of the initial wealth endowment of the worker. Consistent with previous theoretical results, wages are frontloaded when $a_{0}$ is low. At zero wealth the worker receives a wage equal to 1.42 in the first period. Moreover, $w_{0}$ exceeds one up to a wealth level of 0.82 , the buffer stock of savings in the benchmark model. When $a_{0}>0.82$ the firm's constraint is binding and wages always equal unity. These numbers are repeated in the first row of Table 1.

Table 2 (column 1) shows the fraction of employed individuals with wealth below 0.82 . This fraction equals 1 . In other words, all employed individuals (in the steady state distribution) are at the unconstrained optimum. This property can be explained as follows: Suppose that some agents

\footnotetext{
${ }^{14}$ This corresponds to the current policy in most states in the US, assuming that benefits are not extended (as is typical) in periods of "high unemployment". Since my target rate of unemployment is $6.2 \%$, the baseline calibration is realistic.
} 
are employed and have wealth exactly equal to 0.82 . In employment their wealth will remain constant and they will receive $w_{0}=\bar{w}=y$. However, when they become unemployed they will reduce their asset level since their income will drop. When they become employed again they will have $a_{0}<0.82$. The optimal contract will set $a_{1}>a_{0}$ but also $a_{1}<0.82$. This argument demonstrates that the upper bound of the steady state wealth distribution is strictly less than the buffer stock.

The remaining columns in Table 2 give the average levels of consumption for employed individuals (second column) and unemployed individuals with duration $j=0,1$ (third and fourth columns) and $j \geq 2$ (fifth column). To distinguish from previous notation I denote by $\bar{c}^{e}$ the average consumption of employed agents and by $\bar{c}_{j}^{u}, j \in\{0,1, \geq 2\}$ the analogous objects for unemployed agents. At $j \geq 2$ I pool together all spells with durations exceeding 1 quarter. Note that since all employed individuals are at the unconstrained optimum, we have that $\frac{\bar{c}^{e}}{\bar{c}_{0}^{u}}=\left(1+\frac{\frac{1}{\beta}-r}{r \lambda}\right)$ (for all individuals and for the average). $\bar{c}^{e}$ equals 0.949 and $\bar{c}_{0}^{u}$ 0.791. Moreover, consumption drops during the unemployment spell. We have that $\bar{c}_{1}^{u}=0.52$ and $\bar{c}_{\geq 2}^{u}=0.146$. This is explained by the drop in wealth and the fact that $b_{j}=0, j=2,3, \ldots$

\subsection{Should Unemployment Benefits be Backloaded?}

I now consider the effects of 'backloading' unemployment benefits. I first let benefits be equal to zero at $j=0$ and keep them constant at $0.5 y$ at $j=1$. Thereafter, benefits are equal to zero. The behavior of the model is evaluated in the new steady state.

The results from this policy change are summarized in Tables 1 and 2 (second row) and in Figure 2. In the figure, the crossed (purple) line shows the new wage schedule. The region in which wages are frontloaded is now extended to the right, $w_{0}=1.90$ at $a_{0}=0$ and the buffer stock is at 1.39. From Table 1 we see that taxes drop by roughly 2.7 percentage points (3.3\% vs. 0.66\%), however, the unemployment rate and the fraction of agents which have experienced a duration less than or equal to two quarters $\left(\% u_{\leq 1}\right.$, column 3$)$ do not change. The labor market aggregates are unaffected by the new benefit scheme.

These results can be explained as follows: First, since $w_{0}$ increased, workers now hold more assets when they lose their jobs. The wealth effect reduces the search effort exerted during the first period of unemployment. At the same time, the government provides zero benefits in that period; therefore, the effect from benefits goes in the opposite direction. Higher wealth and lower benefits balance each other, in equilibrium there is no impact on the unemployment rates. ${ }^{15}$

In row 3 of Table $1 \mathrm{I}$ show the effects from a policy which sets $b_{1}=1$ and $b_{0}=0$ (equivalent to postponing benefits relative to the benchmark); The effects are similar; under the new scheme wages become more frontloaded and the buffer stock level of savings increases. The dashed -dotted (black) line in Figure 2 shows the new wage schedule. Notice that now there is an impact on the unemployment rates since this policy increases the resources given to unemployed individuals. ${ }^{16}$ Individuals reduce their search intensity, and the unemployment rate increases.

\footnotetext{
${ }^{15}$ The new wage schedule is roughly 0.5 points higher under the new policy than under the benchmark. Since assets earn zero net return, benefits and wealth are perfect substitutes. Another way of saying this is that with $r=1$ the worker's consumption is not financed through assets. As we have seen, $a_{t}$ remains constant for $t \geq 1$, the worker consumes $\bar{w}-\tau$ in every period. Wealth is only used to provide insurance against unemployment.

16 The net effect is no longer equal to zero; if an individual remains unemployed for at least two periods then the benefits received are the same as under the benchmark. However, $w_{0}$ and assets have increased.
} 
Welfare effects from backloaded benefits. Do individuals gain from these policies? I compute the compensating variation, the percentage increase in consumption needed to make agents willing to remain under the benchmark UI policy. The results are shown in columns 4 to 6 of Table 1. $\widetilde{\mu}$ represents the average increment, $\widetilde{\mu}_{e}$ and $\widetilde{\mu}_{u}$ represent the analogous increments for employed and unemployed individuals respectively. Under the new policies, $\widetilde{\mu}$ equals roughly $-0.40 \%$. Agents are willing to give up 0.40 percent of consumption to remain in the old regime. I get similar numbers for employed and unemployed agents separately.

To understand this result consider again the numbers reported in Table 2. From the second and third rows we see that 'backloading benefits' decreases average consumption in employment. This may seem surprising since taxes drop, and as discussed previously, when $r=1$ we have $c_{t}^{e}=\bar{w}-\tau$ in the unconstrained optimum. However, $\bar{w}$ decreases as now agents borrow more to insure against unemployment. This effect, together with the fact that in the model most individuals are employed, explains the welfare patterns.

The above results are robust across different specifications. For brevity, I considered only two policies here. However, I obtain very similar results under any $b_{1}$ maintaining $b_{0}=0$. Backloading benefits increases the cost of unemployment insurance through shifting the 'insurance mix' away from public insurance and towards private insurance. This imparts a negative welfare effect on the population.

\subsection{Should Unemployment Benefits be Permanent?}

Let me now assume that the government runs the following scheme: $b_{j}=0.5 y$ for $j=0,1,2 \ldots$ In other words, unemployment benefits last forever. This type of policy has been analyzed theoretically in many papers in the literature and therefore is worth considering here.

The effects are shown in Tables 1 and 2 (row 4). The new policy reduces the amount of firm insurance. Wages drop and the new buffer stock of savings is 0.65 . Table 1 shows the welfare impact. We have $\widetilde{\mu}=0.19 \%$. On average individuals gain from the new policy. This also holds separately for employed and unemployed individuals. ${ }^{17}$

From Table 2 we see that average consumption is lower relative to the benchmark: we have that $\bar{c}^{e}=0.945$ vs. 0.949 in the benchmark. ${ }^{18}$ It may therefore seem surprising that permanent benefits improve welfare for all agents. This happens because they provide insurance against long term unemployment. Since the terms $U(a, j), j>0$ are discounted by the employed agents, they also gain from the policy change. Insuring agents against long durations of unemployment is, therefore, an important objective of the government.

\subsection{Should Unemployment Benefits be Frontloaded?}

I now set $b_{0}>b_{j}$ for $j=1,2, \ldots$ In Tables 1 and 2 , I study two such policies: First, I set $b_{0}=1, b_{j}=0$ and second, I set $b_{0}=1 b_{j}=0.5$. Under both cases the UI scheme decreases firm insurance. As

\footnotetext{
${ }^{17}$ In Figure 4 in the online appendix I study the welfare impact of different levels of permanent benefits. I find that i) individuals gain as long as $b_{j}>0.3$ and $b_{j}<0.65$ and ii) the optimum level of benefits is close to 0.5 .

${ }^{18}$ Again it holds that $c_{t}^{e}=\bar{w}-\tau$. Taxes increase under permanent benefits and the average $\bar{w}$ drops since now individuals run down their wealth faster in unemployment (they can rely on benefits to finance consumption). This property is echoed on the consumption levels $\bar{c}_{1}^{u}=0.569$ and $\bar{c}_{\geq 2}^{u}=0.501$. Because of this property, many agents start their new jobs with wealth close to zero, and borrow more from their employers.
} 
Table 1 shows, under the second scheme, we get $w_{0}=1.03$ at $a_{0}=0$ and the buffer stock equals 0.08 .

The large response of firm insurance to the new policies can be explained as follows: Since firms can insure their workforce only against short term unemployment, frontloading benefits has a large direct effect on the private insurance arrangement; $b_{0}$ impacts directly the utility $U(a, 0)$ and decreases the derivative $U_{a}\left(a_{t}, 0\right)$. To maintain the ratio $\frac{c_{t}^{e}}{c_{t}^{u}}=\left(1+\frac{\frac{1}{\beta}-r}{r \lambda}\right)$ the firm must now promise to pay a higher level of $c_{t}^{e}$ at the unconstrained optimum. This is unsustainable over a long horizon. The firm's constraint binds and as we previously saw, consumption drops over time. This argument explains why now, over a much wider wealth region, the firm's constraint is binding.

Table 2 shows the average consumption levels. Consider first the properties of consumption in unemployment. The new policies give levels $\bar{c}_{0}^{u}=0.808$ and 0.872 , higher than any scheme previously studied. The fraction of individuals who are at the unconstrained optimum equals $76 \%$ in the case of the first policy and $0 \%$ under the second policy. The ratios $\frac{\bar{c}^{e}}{\bar{c}_{0}^{u}}$ equal 1.179 and 1.077 respectively. ${ }^{19}$ This is considerably lower than $1+\left(\frac{1}{\beta}-1\right) \frac{1}{\lambda} \approx 1.2$ under the baseline parameters.

The unemployed have to save. To have a substantial fraction of individuals whose wealth endowment is large enough so that in equilibrium the firms' constraints bind, it does not suffice to lower the buffer stock; it is also important that unemployed individuals want to save, at least in some periods, and their target level of savings exceeds the buffer stock of employed agents. This is indeed a property of 'frontloaded benefits': unemployed individuals want to save at $j=0$ because they know that benefits will drop in the following periods.

To illustrate this, in Figure 3 I show the savings schedules as functions of wealth. I plot many cases in the figure; the top left panel corresponds to the benchmark model, the top right shows the case of permanent benefits and the bottom panels show the two frontloaded schemes. The solid (blue) lines show savings for employed agents, the dashed (red) lines for unemployed agents at $j=0$. The dashed-dotted (black) lines are at 45 degrees.

Consider the bottom panels, left and right. These show clearly that the buffer stock of savings of employed agents corresponds to a lower wealth level than the buffer stock of unemployed agents. In the bottom right for example, we get 0.08 and 0.71 for employed and unemployed individuals respectively. Unemployed agents save considerably during the first quarter; then, with a large probability, they return to employment and their wealth level already exceeds the buffer stock of the employed. This happens only when benefits are frontloaded.

Welfare under frontloaded benefits. Columns 4-6 of Table 1 present the welfare effects of 'frontloading' benefits. There are substantial gains for both unemployed and employed individuals. Paying benefits equal to 1 only during the first period of unemployment, yields a compensating variation coefficient of $0.2 \%$, the analogous number in the case of the scheme $b_{0}=1 b_{j}=0.5, j>0$ is $54 \%$ ( $51 \%$ for employed agents and $98 \%$ for the unemployed). The second policy performs better than the first one because it produces a smaller drop in consumption during unemployment.

\footnotetext{
19 The average consumption of employed individuals equals roughly 0.95 under the first scheme and 0.94 under the second (Table 2). Both numbers exceed $y-\tau$. As we have seen, when the firm's participation constraint binds, consumption drops over time. It equals $y-\tau$ in the limit, when wealth converges to the buffer stock.
} 


\subsection{Welfare Maximizing Benefit Scheme}

I now turn to the evaluation of the benefit scheme which maximizes welfare. I consider a restricted class of policies here, in particular policies which pay out different levels of benefits over three time intervals: the government pays $b_{0}$ to the newly unemployed, $b_{1}$ to those who have already been unemployed for one quarter, and $b_{\geq 2}$ for durations exceeding two quarters. I maximize welfare over $b_{0}, b_{1}$ and $b_{\geq 2}$.

I restrict the analysis along these lines for two reasons: First, because in the model most spells end after two quarters. Therefore, extending the analysis to more complex benefit schemes should not affect my conclusions. Second, because with too few agents experiencing durations longer than three quarters it is computationally very difficult to maximize welfare with respect to the benefit level in the fourth, the fifth quarter and so on; the optimal policy is close to indeterminate in these cases. Given these remarks, I suspect that the results I present in this paragraph are close to 'optimal' in the sense that if the government were allowed to chose a longer sequence of $b_{j}, j=0,1,2,3, \ldots$ the implications that I derive here would not be affected.

The welfare maximizing policy is the following: $b_{0}$ equals $1.55, b_{1}$ equals 0.1 and finally, $b_{\geq 2}=0.25$. With these benefit levels the gain relative to the baseline UI scheme is $0.687 \%$. For employed individuals it is $0.63 \%$ and for unemployed individuals $1.374 \% .^{20}$

The last rows of Tables 1 and 2 summarize the properties of the model. There are several noteworthy features: From Table 1, the buffer stock of savings for employed individuals is equal to zero. This means that when the worker becomes employed, it is optimal to reduce her stock of savings until the borrowing constraint is hit. From Proposition 5 we know that the firm's participation constraint always binds and wages equal $y$. No agents receive a frontloaded wage in equilibrium.

Remarkably, under the welfare maximizing scheme, average consumption increases when agents become unemployed. I obtain $\frac{\bar{c}^{e}}{\bar{c}_{0}^{u}}=\frac{0.940}{0.952} \approx 0.987$. But because benefits decrease sharply, consumption drops fast in subsequent periods. Since workers suffer with positive probability a drop in consumption at $j=1,2, \ldots$ it still holds that $W(a, 0)>U(a, 0)$ (e.g. A.2).

\section{Extensions and Robustness}

\subsection{The Effects of Interest Rates}

The analysis of the previous sections illustrated that when the cost of borrowing from the firm is large, in an unconstrained optimum the worker suffers a big drop in consumption during the first period in unemployment. With the benchmark parameters this drop was in the order of $17.3 \%$. The smaller the difference between $\frac{1}{\beta}$ and $r$, the smaller this loss of consumption is. I now relax the assumption that workers earn zero return on their savings. I let the net rate of return $r-1$ be equal to $0.5 \%$ (giving an annual return of roughly $2 \%$ ). I keep $\beta$ at its previous level. With the new parameters $1+\frac{\frac{1}{\beta}-r}{r \lambda} \approx 1.099$.

Under the new calibration, I repeat the numerical experiments of the previous section. For brevity

\footnotetext{
${ }^{20}$ I leave for future work to study the welfare effects in the transition. The reader may have noticed that switching to the 'optimal scheme' from the benchmark, may imply that participation is not satisfied for some individuals in the economy. Agents which begun their jobs with low $a_{0}$ under the old regime and chose a high $J_{1}=J_{2}=\ldots$ may find that now with higher benefits it is preferable to quit their jobs. This clearly cannot happen in steady state.
} 
the results are summarized in the online appendix (see Tables 4 and 5). There are several features worth highlighting here: First, under all UI schemes considered $w_{0}$ and the buffer stock of savings increase, since the drop in consumption in unemployment is smaller. Second, the distribution of welfare gains and loses across UI schemes is somewhat different than under the benchmark calibration. Whereas policies which backload benefits continue to produce welfare loses, these are now smaller in magnitude. ${ }^{21}$ Moreover, the constant benefit policy no longer imparts a positive welfare effect to the population $(\widetilde{\mu}=-0.20 \%$ for all agents).

To understand these findings note first that 'backloading benefits' becomes less harmful owing to the fact that the cost of private insurance is now smaller. It is less inefficient to let firms insure their workers against unemployment, government insurance no longer corrects a large inefficiency when it crowds out private insurance. Second, in cases where the government pays positive benefits forever, the cost from distortionary taxation offsets any gain from crowding out private insurance. Third, constant benefit policies are now less beneficial because assets earn a higher return; when the agent is unemployed her consumption drops at a slower pace when $r>1$. Unemployment insurance becomes less necessary to finance consumption over long durations of unemployment. ${ }^{22}$

The optimal UI scheme is as follows: $b_{0}=1.05$ and $b_{1}=b_{\geq 2}=0$. The compensating variation coefficient equals $0.29 \%$. In the steady state a small fraction of individuals (38\%) are at the unconstrained optimum, the remaining agents have assets which exceed the buffer stock of savings. This makes the ratio $\frac{c^{e}}{c^{u}} \approx 1.083$ slightly lower than 1.099. The scope of government provided unemployment insurance when the interest rate is higher is clearly more limited, however, the conclusion that unemployment benefits should be frontloaded remains.

\subsection{Benefits under flat wage contracts}

In now turn to the properties of the welfare maximizing policy in the heterogeneous agents model. Note that though the optimal policy under limited commitment (when $r=1$ ) lead essentially to a 'heterogeneous agent outcome' it is not obvious that the two models produce the same welfare maximizing policy. In the heterogeneous agent model wages are flat under all UI schemes, under limited commitment they are not. The government faces different tradeoffs, this may give rise to different outcomes in terms of policy.

In the model with $r=1 \mathrm{I}$ obtain the following 'optimal' policy: $b_{0}=1.6389 b_{1}=0.1444$ and $b_{\geq 2}=0.3667$. Moreover, when I assume that $r-1=0.5 \%$ I get $b_{0}=b_{1}=b_{\geq 2}=0$ (i.e. it is optimal to have no public unemployment insurance at all). In both cases the welfare gains from switching to the welfare maximizing policy are substantial. I obtain values for the compensating variation equal to 0.4649 and 0.3788 respectively.

To explain the driving force behind these results it is important to remember that the two models impart a very different behavior on savings. Under heterogeneous agents (precautionary) savings yield a consumption level for the employed individual which increases over time (until the stationary buffer stock is built). In that model the cost of private insurance is measured in terms of the rising consumption path. Under the optimal contract, asset accumulation is frontloaded and the stationary

\footnotetext{
${ }^{21}$ For example, $\widetilde{\mu}=-0.22 \%$ when $b_{1}=0.5$ and $b_{j}=0, j \neq 1$.

${ }^{22}$ See for example the average consumption patterns at $j=1$ and $j \geq 2$ documented in Table 5 in the appendix. See also Young (2004) for a discussion of this effect in general equilibrium in the standard heterogeneous agents model.
} 
stock is built in one period; the cost of insurance in this case is the interest rate cost on the loan given from the firm to the worker.

When $r=1$ both models clearly yield a substantial cost of private insurance: When the interest rate in the market is low, the cost of accumulating precautionary savings is high, as the optimal consumption path would be decreasing (at rate $\beta$ ) if the government eliminated the risk of unemployment. Therefore, in both cases the government has the incentive to completely crowd out asset accumulation. When $r$ increases the costs of insurance are mitigated in both models.

To conclude, the optimal policy is similar between the limited commitment model and the heterogeneous agent model when $r=1$. However, it is different when $r>1$. The differences between the two models are worth to be separately considered in future work.

\subsection{Decentralizing through Severance Payments}

One of the goals of this paper was to extend the limited commitment model of wages to endogenous savings. A key prediction of the model is that wages may be frontloaded. Though the idea that wages are paid upfront may seem odd, the allocation predicted by the model can be decentralized into a constant wage and severance payment scheme. This for example is the case if we assume that the difference $a_{1}-a_{0}$ is set aside and given to the worker when she becomes unemployed. ${ }^{23}$ Since severance payments are a very common private insurance arrangement in the US (see for example Chetty (2008) and Pissarides (2010)) the wage profile predicted by the model becomes a reasonable approximation of the data.

Given the link between firm insurance and severance compensation in the model, it is interesting to investigate whether current policies in the US discriminate between cases where workers receive severance and cases where they do not. To answer this question data were collected from different states to obtain information on the treatment of severance payments by the state authorities. ${ }^{24}$ Table 3 summarizes the information. There are basically two types of policies followed: in the first column I list states in which if the applicant has received severance payments, benefits are reduced by the weekly prorated amount of these payments. Under column (2) I show the remaining states, in which benefits are either reduced only in the week the severance payment is received, or they are not reduced at all. Approximately, I think of column (1) states, as states where benefits are foregone in the presence of private risk sharing and column (2) states, as states where benefits are not contingent on the receipt of private insurance. ${ }^{25}$

\footnotetext{
${ }^{23}$ The simplest case to visualize this is $r=1, c^{e}=\bar{w}-\tau$ and assets are not used to finance consumption during employment. Then, we can let $w_{t}=\bar{w}$ for all $t$. The difference $a_{1}-a_{0}$ represents the severance payment.

In the online appendix I show that the optimal allocation when the worker chooses assets and severance payments, can indeed be interpreted as a frontloaded wage profile. Though this is a general result, to obtain the exact profile I had in the previous sections, I need to introduce a few frictions to the model. I assume that i) the firm can make positive profits if severance payments are positive and ii) the worker does not internalize the effect of her choices on the firm's profits. These are two simple modifications of the problem, possibly not the only ones I could have used. They are important since when $R>r$ severance payments modify the constraint set and the higher rate $R$ creates wealth intertemporally. See Pissarides (2010) for a deeper microfoundation of why severance payments do not provide complete insurance.

${ }^{24}$ The data were collected from the web and through phone interviews with personnel in the relevant agencies. Three states are missing because the web did not provide any information concerning the treatment of severance compensation and the phone numbers listed were not responding.

25 Since one week is a small fraction of the horizon over which unemployment benefits are paid (6 months) I treat this as a case as no reduction of benefits. Moreover, in all states in which benefits are reduced by the prorated amount, they are typically foregone. The worker is not entitled to unemployment benefits if her spell exceeds 6 months. The
} 
Should benefits be contingent on severance payments as is the case in Colorado, or should they complement private insurance as in California? I considered policies which eliminated benefits during the first quarter of unemployment. Approximately this can be thought as reducing unemployment benefits when workers receive firm insurance. ${ }^{26}$ When I set $b_{0}=0$ private insurance was crowded in. However, my simulations showed that workers strictly prefer not to have $b_{0}=0$. The reason is that private risk sharing is more costly (and less efficient) than public insurance.

\section{Conclusions}

This paper studies the effects of unemployment benefits, in an economy where firms offer to their workforce a contract subject to limited commitment. It is shown that when workers have access to a storage technology, savings are utilized to provide (partial) insurance against the risk of a job separation. The participation constraints implied by limited commitment influence the scope of risk sharing. In this environment the government has the explicit goal to drive the allocation to the point where the firm's participation constraint is binding. I illustrate that at this point the drop in consumption suffered by the worker when she becomes unemployed is smaller. The welfare maximizing UI scheme entails large frontloaded benefits.

A number of extensions are worth considering. First, it was assumed that matches terminate through exogenous job destruction shocks. This has left out of the analysis match quality shocks. It is worth exploring how these additional sources of risk may impact the optimal contract in the presence of savings. This is not trivial; since assets break the link between consumption and wages, wages may become very volatile without resulting in excess volatility of consumption. It would be interesting to investigate whether commitment affects the variability of wages in the way that the existing literature has identified. Moreover, job retention effort (e.g. Wang and Williamson (2002)) could be an important addition to the framework. When worker effort determines the probability of a separation, the optimal contract trades-off insurance against retention in the presence of a potent wealth effect. Modelling this tradeoff in the case where effort is not observable by the firm and studying the effects of unemployment benefits, adds another important and interesting layer to the analysis presented.

\section{References}

Aiyagari, R. (1994) "Uninsured Idiosyncratic Risk and Aggregate Saving," Quarterly Journal of Economics, 109 (3): 659-684

Attanasio, O., and Rios Rull, J (2000) "Consumption Smoothing in Island Economies: Can Public Insurance Reduce Welfare?" European Economic Review 44 (7): 1225-1258.

exception to this rule is the state of Arizona.

${ }^{26} \mathrm{As}$ I showed, in the benchmark model and in the models with backloaded benefits, all individuals get firm insurance. Hence eliminating unemployment benefits for all agents is equivalent to eliminating them for agents which receive firm insurance. 
Chetty, R. (2008) "Moral Hazard vs Liquidity in Optimal Unemployment Insurance "Journal of Political Economy, 116 (2): 173-234.

Deaton, A. (1991) "Saving and Liquidity Constraints," Econometrica, 59 (5): 1221-1248.

Gruber, J. (1997): "The Consumption Smoothing Benefits of Unemployment Insurance," American Economic Review, 87(1): 192-205.

Hansen, G. and Imhrohoroglu, A. (1992) "The Role of Unemployment Insurance in an Economy with Liquidity Constraints and Moral Hazard," Journal of Political Economy, 100 (1): 118-142.

Huggett, M. (1993) "The Risk Free Rate in Heterogeneous Agents Incomplete Insurance Economies, " Journal of Economic Dynamics and Control, 17: 953-969.

Karaivanov, A., and Martin, F. (2015) "Dynamic Optimal Insurance and Lack of Commitment " Review of Economic Dynamics, 18 (2): 287-305.

Kocherlakota , N. (1996) "Implications of Efficient Risk Sharing Without Commitment ," Review of Economic Studies, 63 (4): 595-609

Krusell, P., Mukoyama, T. and Sahin , A. (2010) "Labour Market Matching with Precautionary Savings and Aggregate Fluctuations," Review of Economic Studies, 77 (4): 1477-1507.

Ligon, E., Thomas, J., and Worrall, T. (2000) "Mutual Insurance, Individual Savings and Limited Commitment," Review of Economic Dynamics, 3 (2): 216-246.

Ligon, E., Thomas, J., and Worrall, T. (2002) "Informal Insurance Arrangements with Limited Commitment: Theory and evidence from village economies," Review of Economic Studies, 69 (1): 209-244.

Ljungqvist, L. and Sargent, T. (2000) "Recursive Macroeconomic Theory,", 2nd Edition MIT Press, Cambridge MA.

Marcet, A. and Marimon, R. (2002) "Recursive Contracts,", mimeo

Nakajima, M. (2012) "A Quantitative Analysis of Unemployment Benefits Extensions,", Journal of Monetary Economics, 59 (7): 686-702.

Oikonomou, R. (2010). "Essays in Incomplete Insurance and Labor Markets with Frictions ,"PhD Thesis, London School of Economics.

Pissarides, C. (2010) "Why Do Firms Offer Employment Protection, "Economica, 77 (308): 613636.

Rudanko , L. (2009) "Labor Market Dynamics Under Long Term Wage Contracting," Journal of Monetary Economics, 56 (2): 170-183.

Rudanko, L. (2011) "Aggregate and Idiosyncratic Risk in a Frictional Labor Market," American Economic Review, 101 (6): 2823-43. 
Thomas, J. and Worrall, T. (1988) "Self-Enforcing Wage Contracts," Review of Economic Studies, 55 (4): $541-554$.

Thomas, J. and Worrall, T. (2007) "Unemployment Insurance Under Moral Hazard and Limited Commitment: Public Versus Private Provision," Journal of Public Economic Theory, 9(1): 151181.

Wang. C., and Williamson, S. (2002) "Moral Hazard, Optimal Unemployment Insurance and Experience Rating," Journal of Monetary Economics, 49 (7): 1337-71.

Voena, A. (2015) "Yours, Mine and Ours: D Divorce Laws Affect the Intertemporal Behavior of Married Couples," American Economic Review, 105 (8): 2295-2332

Young, E. (2004) "Unemployment Insurance with Capital Accumulation," Journal of Monetary Economics, 51 (8): 1683-1710. 


\section{A Appendix}

\section{A.1 Competitive Equilibrium}

The (stationary) competitive equilibrium consists of a set of value functions $\{U(a, j), W(a, J)\}$ for unemployed and employed workers respectively, a set of decision rules on asset holdings $\left\{a_{e}^{\prime}(a, J), a_{u}^{\prime}(a, j)\right\}$, the firm's continuation value $J^{\prime}(a, J)$, and the search intensity rule $s(a, j)$. It also consists of a level of taxes $\tau$ and an invariant measure $\mu$ of agents across assets, employment status and $J$ such that:

1) Agents optimize: $\{U(a, j), W(a, J)\}$ solve functional equations (2) and (6) and optimal policies derive.

2) Taxes and benefits are consistent with budget balance: $e \tau=\sum_{j<m} u_{j} b_{j}$

3) The measure $\mu$ is consistent: In particular the law of motion of $\mu$ can be represented as:

$$
\begin{gathered}
\mu_{(e, \mathcal{A}, \mathcal{J})}=(1-\lambda) \int_{\left(a^{\prime}(e, a, J) \in \mathcal{A}\right) \cap\left(J^{\prime}(a, J) \in \mathcal{J}\right)} d \mu_{(e, a, J)}+\mathcal{I}_{(0 \in \mathcal{J})} \sum_{j} \int_{a_{u}^{\prime}(a, j) \in \mathcal{A}} \gamma(s(a, j)) d \mu_{(u, a, j)} \\
\mu_{(u, \mathcal{A}, j)}=\mathcal{I}_{(j=0)} \lambda \int_{a_{e}^{\prime}(a, J) \in \mathcal{A}} d \mu_{(e, a, J)}+\mathcal{I}_{(j>0)} \int_{a_{u}^{\prime}(a, j-1) \in \mathcal{A}}(1-\gamma(s(a, j-1))) d \mu_{(u, a, j-1)}
\end{gathered}
$$

where $\mathcal{A}$ and $\mathcal{J}$ are subsets of the relevant state space and $\mu_{(u, \mathcal{A}, j)}, \mu_{(e, \mathcal{A}, \mathcal{J})}$ are the probability distributions conditional on employment status. $\mathcal{I}_{(x)}$ is an indicator variable which takes the value one if $x$ is true and zero otherwise. ${ }^{27}$

Proof of Proposition 2 Suppose that we have $\phi_{0}=0$ and $\phi_{T}>0$ for some T. From (7) and (8) we have that: i) $c_{0}^{e}=c_{1}^{e}=\ldots=c_{T}^{e} \equiv \bar{c}^{e}$ and $\frac{c_{t}^{e}}{c_{t}^{u}}=1+\frac{\left(\frac{1}{\beta}-r\right)}{r \lambda} \equiv \kappa$ for $t=1,2, \ldots T$.

Note that if the firm's constraint first binds in period $T$ we have that $J_{1}, J_{2}, \ldots J_{T}>0$ and $J_{T+1}=0$. From the optimality conditions we can show that $J_{1}=J_{2}=\ldots=J_{T}>0$. Suppose the converse; note that from $c_{t-1}^{e}=c_{t}^{e}$ and $\frac{c_{t-1}^{e}}{c_{t-1}^{u}}=\frac{c_{t}^{e}}{c_{t}^{u}}$ we have that consumption and assets are constant. From the agent's budget constraint it must also be that wages are constant. From the promise keeping constraints it is trivial to show that the $J s$ are constant.

Given this property we can write $\left(J_{0}, J_{1}, \ldots, J_{T}, J_{T+1}\right)=(0, \bar{J}, \ldots, \bar{J}, 0) . J$ starts at zero, subsequently remains to some constant value $\bar{J}>0$ for $T$ periods and then drops to 0 . The sequence of wages between period 0 and $T$ satisfies: $\left(w_{0}, w_{1}, \ldots, w_{T-1}, w_{T}\right)=(y+\beta(1-\lambda) \bar{J}, y-(1-\beta(1-$ $\lambda)) \bar{J}, \ldots, y-(1-\beta(1-\lambda)) \bar{J}, y-\bar{J})$.

To simplify assume wlog that $r=1$ and $\tau=0$. We have that $\bar{c}^{e}=y-(1-\beta(1-\lambda)) \bar{J}$. Moreover, $a_{1}=a_{2}=\ldots=a_{T}=a_{0}+\bar{J} \equiv \bar{a}$. Let $\widetilde{a}$ denote the level of assets in $T+1$; we obtain: $\widetilde{a}=\bar{a}-\beta(1-\lambda) \bar{J}$. In $T+1$ assets drop since wages drop in $T$.

To show that the worker cannot optimize and choose $\left(a_{T+1}, J_{T+1}\right)=(\widetilde{a}, 0)$ I proceed in two steps. First, I assume that there is a unique optimum in terms of the policy rule which solves the value function. Otherwise, if the agent is indifferent between $(\bar{a}, \bar{J})$ and $(\widetilde{a}, 0)$ in period $T$, she will also be indifferent in period 0 as will become evident subsequently. ${ }^{28}$ We can now rule out $T>1$ through claiming that $(\widetilde{a}, 0)$ is obviously inconsistent with this worker's optimization. Notice that in $T-1$ we have:

$$
W(\bar{a}, \bar{J})=\log \left(\bar{c}^{e}\right)+\beta(\lambda U(\bar{a}, 0)+(1-\lambda) W(\bar{a}, \bar{J}))>\log \left(\bar{c}^{e}\right)+\beta(\lambda U(\widetilde{a}, 0)+(1-\lambda) W(\widetilde{a}, 0))
$$

since the vector $\left(\bar{c}^{e}, \widetilde{a}, 0\right)$ is in the constraint set in $T-1$. However, in $T$ we have that

$$
W(\bar{a}, \bar{J})=\log \left(\bar{c}^{e}\right)+\beta(\lambda U(\widetilde{a}, 0)+(1-\lambda) W(\widetilde{a}, 0))>\log \left(\bar{c}^{e}\right)+\beta(\lambda U(\bar{a}, 0)+(1-\lambda) W(\bar{a}, \bar{J}))
$$

\footnotetext{
${ }^{27}$ For brevity I use the same number of arguments in $\left.\mu_{(e, ., .}\right)$ and $\left.\mu_{(u, ., .}\right)$. The third argument however is $J$ (promised utility) if the agent is employed, and $j$ (duration of the spell) if she is unemployed.

${ }^{28}$ Generally, proving that the optimal policy is single valued is not possible in the commitment problem (see LTW). One has to rely on simulations of the model to verify that the value function is strictly concave. This property holds in my numerical solution.
} 
In other words, the agent solved the same program in $T-1$ as in $T$ and with exactly the same state variables. Unless she is indifferent between the two solutions we have a contradiction.

This contradiction is not obvious in the case where $T=1$ (since now $W\left(a_{T-1}, J_{T-1}\right)=W\left(a_{0}, 0\right)$ ). To show the problem in this case note that since $\phi_{0}=0$ we have that:

$$
W\left(a_{0}, 0\right)=\log \left(\bar{c}^{e}\right)+\beta(\lambda U(\bar{a}, 0)+(1-\lambda) W(\bar{a}, \bar{J}))>\log \left(\bar{c}^{e}\right)+\beta(\lambda U(\widetilde{a}, 0)+(1-\lambda) W(\widetilde{a}, 0))
$$

Again $(\widetilde{a}, 0)$ is affordable in period zero since $\widetilde{a}=a_{0}+y-\bar{c}^{e}$. Now consider the agent's program in period 1 . The agent has two choices: i) to remain in the unconstrained optimum (i.e. $\left(a_{2}, J_{2}\right)=(\bar{a}, \bar{J})$ ) ${ }^{29}$, ii) to choose $\left(a_{2}, J_{2}\right)=(\widetilde{a}, 0)$. In either case consumption in period one must equal $\bar{c}^{e}$ because of (7). Assume that ii) is preferred over i). Then we have:

$$
\log \left(\bar{c}^{e}\right)+\beta(\lambda U(\widetilde{a}, 0)+(1-\lambda) W(\widetilde{a}, 0))>\log \left(\bar{c}^{e}\right)+\beta(\lambda U(\bar{a}, 0)+(1-\lambda) W(\bar{a}, \bar{J}))
$$

which contradicts (11). Hence, i) is preferable to ii).

Since the program is recursive it is trivial to generalize the above to have that if $\phi_{t}=0$ then $\phi_{t+j}=0$ for $j>0$.

Proof of Proposition 4 To prove that the optimal contract is a flat contract insofar as the firm's constraint is binding, I show here that when the constraint binds it binds forever. In other words, the only possibility is to have $T=\infty$ (where $T$ is the last period that the constraint binds in proposition 4). Then, it is trivial to show that $w_{t}=y \forall t$. This can be immediately seen from the firm's promise keeping constraint and the fact that $J_{t}=0 \forall t$.

Assume that $T<\infty$. We have that $\phi_{t}>0$ for $t=0,1,2, \ldots, T-1$ but then $\phi_{T}=0$ and the firm's constraint never binds again after $T+1 .{ }^{30}$ From the promise keeping constraint of the firm we can show that $w_{0}=w_{1}=\ldots=w_{T-1}=y$. From Proposition 1 we know that subsequently we have $w_{T} \geq y$ and $w_{t}=\bar{w} \leq y, t=T+1, T+2, \ldots$.

From the proof of Proposition 1 we know that if the constraint ceases to bind the worker's wealth follows $a_{T} \leq a_{T+1}=a_{T+2}=\ldots=\widetilde{a}\left(a_{T}<a_{T+1}\right.$ if $\left.w_{T}>y\right)$ and $J_{T+1}=J_{T+2}=\ldots=\bar{J} \geq 0$. Before period $T$, since the firm's constraint binds, assets and consumption drop over time (e.g. (7)). Therefore $a_{0}>a_{1}>\ldots>a_{T}$ and $c_{0}^{e}>c_{1}^{e}>\ldots>c_{T}^{e}=c_{T+1}^{e}=\ldots$. Consumption drops over time and remains constant from period $T$ onwards.

From equation (8) for $t \leq T$ and $t>1$ we have that

$$
\frac{c_{t}^{e}}{c_{t}^{u}}=1+\frac{\left(\frac{1}{\beta}-r\right)}{r \lambda}-\frac{1}{(1-\lambda)} \frac{1}{r\left(\lambda \beta^{2}\right)} \phi_{t-1}^{2} c_{t}^{e}<1+\frac{\left(\frac{1}{\beta}-r\right)}{r \lambda}=\frac{c_{T+1}^{e}}{c_{T+1}^{u}}=\frac{c_{T+2}^{e}}{c_{T+2}^{u}}=\ldots
$$

Note that according to the above equation, it holds that $\frac{c_{T}^{e}}{c_{T}^{u}}<\frac{c_{T+1}^{e}}{c_{T+1}^{u}}$. Since $c_{T}^{e}=c_{T+1}^{e}$ this implies that $c_{T}^{u}>c_{T+1}^{u}$. This contradicts the assumption that $a_{T} \leq a_{T+1}$ from the fact that $\frac{r}{c_{t}^{u}}=U_{a}\left(a_{t}, 0\right)$. This path is therefore not consistent with the worker's optimization. There cannot be any $T<\infty$ such that the firm's constraint ceases to bind.

The optimality conditions hold only when $T=\infty$ (put differently, when the firm's constraint always binds). The fact that the constraint always binds should not be surprising. In standard incomplete market models which feature a buffer stock savings, the level of assets which defines the buffer stock is reached in the limit. Here the optimality conditions suggest that the contract features an analogous property. Assets are reduced by smaller and smaller amounts, and in the limit the firm's constraint becomes slack.

\footnotetext{
${ }^{29}$ If the agent chooses the unconstrained optimum she must set $a_{2}=\bar{a}$ since $\bar{c}^{e}=\kappa c_{2}^{u}=\frac{r}{U_{a}\left(a_{2}, 0\right)}=$ $\frac{r}{U_{a}\left(a_{1}-\bar{c}^{e}+y+\beta(1-\lambda) \bar{J}, 0\right)}$. This nonlinear equation gives the same solution for $\bar{c}^{e}$ since $U_{a}$ is monotonically decreasing in assets.
}

${ }^{30}$ As discussed, if a constraint ceases to bind, then it never binds again (e.g. Proposition 2). 
Figure 1: Asset Accumulation Profiles

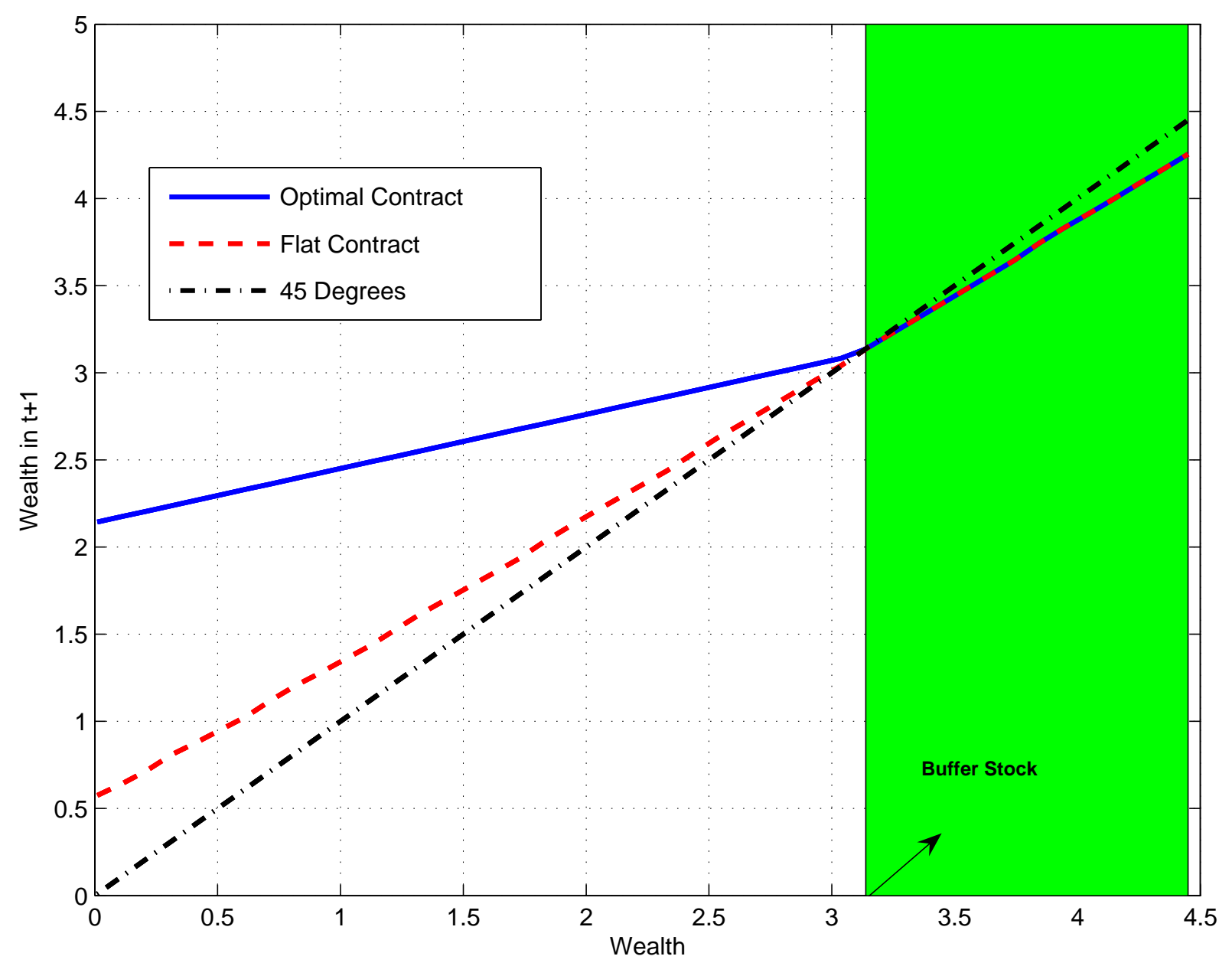

Notes: The solid line shows the asset accumulation rule. The shaded area plots the range of assets over which the firm's participation constraint binds. The $\mathrm{x}$ axis denotes the initial wealth endowment of the worker. The asset accumulation rule applies only in the first period over the range of wealth in the non-shaded area. From period two onwards assets are constant, in the unconstrained optimum, hence the rule becomes equal to the 45 degree line.

The dashed line shows the asset rule in the case of the flat contract (e.g. the solution to equation (10)). The $\mathrm{x}$ axis now denotes wealth in any period. The dashed-dotted line is at 45 degrees. 
Figure 2: Wage Schedules: Various Models

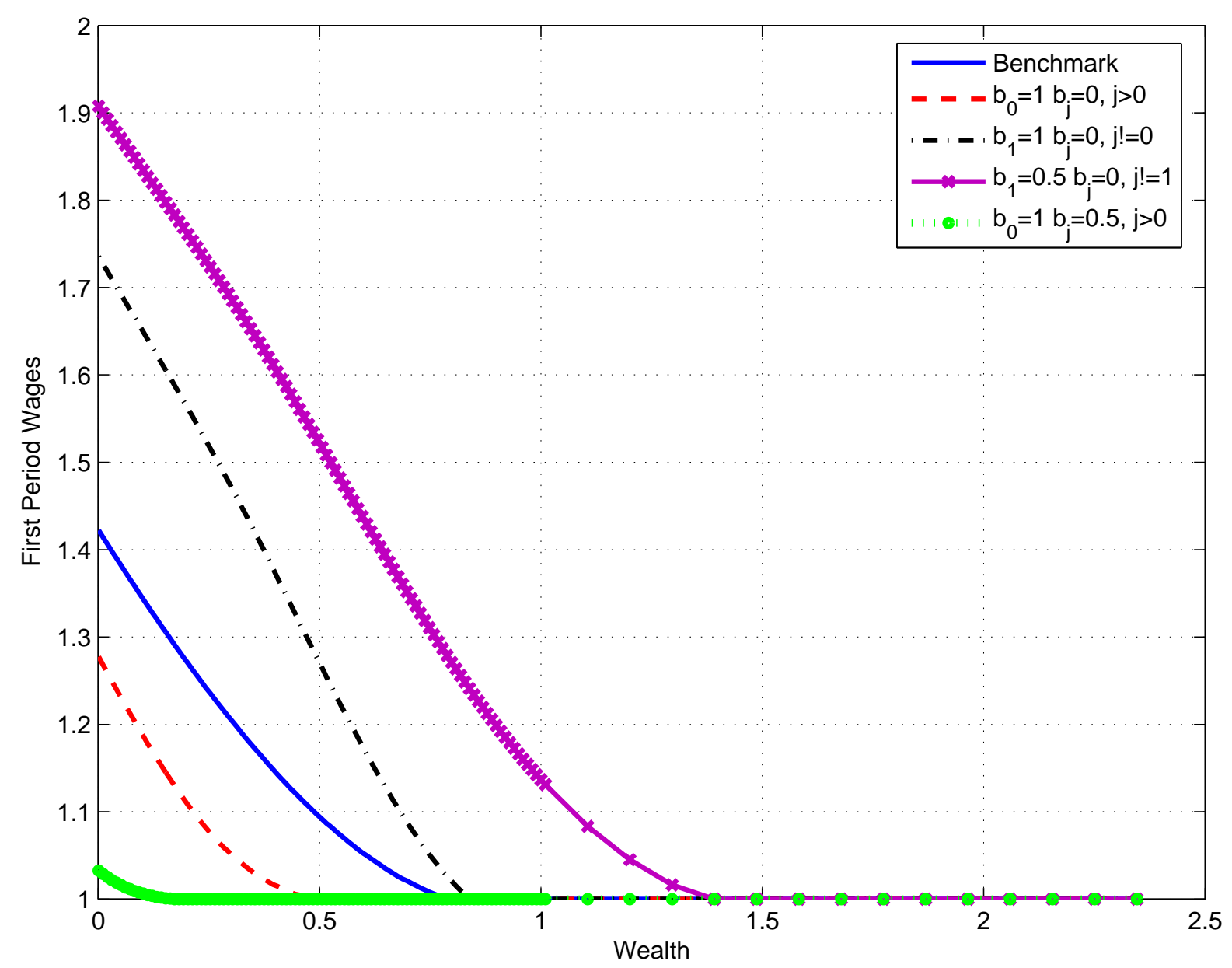

Notes: The figure plots wage schedules (first period wages as a function of initial assets) under various unemployment benefit schemes. The solid (blue) line plots the baseline model, e.g. $b_{0}=$ $b_{1}=0.5$ and $b_{j}=0, j=2,3, \ldots$. The dashed red line shows a frontloaded scheme setting $b_{0}=1$ and $b_{j}=0, j=1,2, \ldots$ The dashed-dotted (black) line sets $b_{1}=1$ and benefits are zero in all other periods. The crossed (purple) line shows a UI scheme which eliminates the first period benefits and pays out 0.5 only in the second period. Finally, the line marked by circles (green) sets $b_{0}=1$ and $b_{j}=0.5, j=1,2,3 \ldots$. 
Figure 3: Savings Schedules in Employment and Unemployment: Various Models
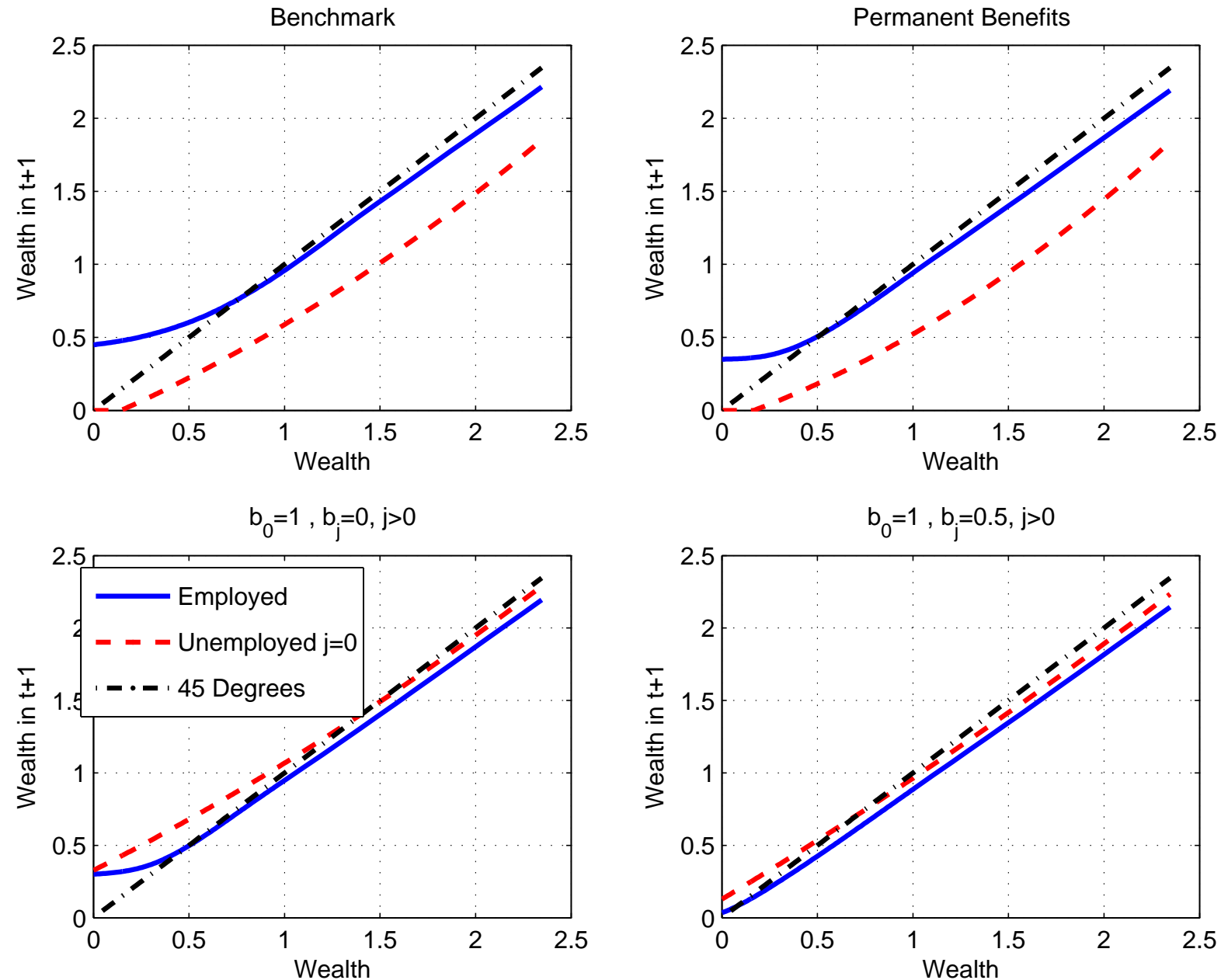

Notes: The figure plots the savings schedules in employment (solid lines, first period of the optimal contract) and in the first period of unemployment (dashed-red lines) under 4 different unemployment benefit schemes. The top panel shows the benchmark model. The top right shows the case of constant permanent benefits. The bottom panels show 'frontloaded benefits'. The bottom left shows benefits $b_{0}=1$ and $b_{j}=0, j \geq 1$ and the bottom right $b_{0}=1$ and $b_{j}=0.5, j \geq 1$. The dashed-dotted (black) lines are at 45 degrees. 
Table 1: Effects of UI Policies: Baseline model $r=1$

\begin{tabular}{lccccccccc} 
& Policy & $\tau$ & $u$ & $\% u_{\leq 1}$ & $\widetilde{\mu}$ & $\widetilde{\mu}_{e}$ & $\widetilde{\mu}_{u}$ & $w_{0}$ & Buffer \\
\hline \hline Benchmark & $b_{0}=b_{1}=0.5$ & $3.30 \%$ & $6.20 \%$ & $96.0 \%$ & 0 & 0 & 0 & 1.42 & 0.82 \\
\multirow{2}{*}{ Backloaded } & $b_{1}=0.5$ & $0.66 \%$ & $6.20 \%$ & $96.1 \%$ & $-0.41 \%$ & $-0.42 \%$ & $-0.40 \%$ & 1.90 & 1.39 \\
& $b_{1}=1$ & $1.81 \%$ & $6.75 \%$ & $94.6 \%$ & $-0.44 \%$ & $-0.45 \%$ & $-0.30 \%$ & 1.74 & 0.85 \\
Permanent & $b_{j}=0.5$ & $3.68 \%$ & $6.86 \%$ & $90.3 \%$ & $0.19 \%$ & $0.18 \%$ & $0.40 \%$ & 1.32 & 0.65 \\
\multirow{2}{*}{ Frontloaded } & $b_{0}=1$ & $5.03 \%$ & $5.94 \%$ & $96.8 \%$ & $0.20 \%$ & $0.20 \%$ & $0.20 \%$ & 1.28 & 0.62 \\
\multirow{2}{*}{ Optimal } & $b_{0}=1 b_{j>0}=0.5$ & $6.23 \%$ & $6.92 \%$ & $89.9 \%$ & $0.53 \%$ & $0.51 \%$ & $0.98 \%$ & 1.03 & 0.08 \\
\hline \hline
\end{tabular}

Notes: The table shows the effects of various UI schemes. $b_{j}$ is the level of benefits given in period $j+1$ of an unemployment spell. $j=0$ corresponds to benefits received during the first quarter, $j=1$ during the second and so on. $b^{*}$ denotes the optimal policy. As discussed in text, we have $b_{0}^{*}=1.55 b_{1}^{*}=0.1 b_{\geq 2}^{*}=0.25$ For the remaining columns the level of unemployment benefits in $j$ is reported when $b_{j}>0$.

$\% u_{\leq 1}$ represents the fraction of unemployed individuals with duration less than or equal to 2 quarters. $\widetilde{\mu}$ is the percentage increment in consumption needed by individuals to be as well off as under the baseline UI policy.

Table 2: Average consumption in employment and unemployment: Baseline model $r=1$

\begin{tabular}{lcccccc} 
& Policy & $\% w_{0}>y$ & $\bar{c}^{e}$ & $\bar{c}_{o}^{u}$ & $\bar{c}_{1}^{u}$ & $\bar{c}_{\geq 2}^{u}$ \\
\hline \hline Benchmark & $b_{0}=b_{1}=0.5$ & $100 \%$ & 0.949 & 0.791 & 0.520 & 0.146 \\
\multirow{2}{*}{ Backloaded } & $b_{1}=0.5$ & $100 \%$ & 0.926 & 0.772 & 0.534 & 0.155 \\
& $b_{1}=1$ & $100 \%$ & 0.943 & 0.786 & 0.663 & 0.245 \\
Permanent & $b_{j}=0.5$ & $100 \%$ & 0.945 & 0.788 & 0.569 & 0.501 \\
\multirow{2}{*}{ Frontloaded } & $b_{0}=1$ & $76 \%$ & 0.953 & 0.808 & 0.478 & 0.122 \\
\multirow{2}{*}{ Optimal } & $b_{0}=1 b_{j>0}=0.5$ & $0 \%$ & 0.940 & 0.872 & 0.680 & 0.501 \\
\hline \hline & $b^{*}$ & $0 \%$ & 0.940 & 0.952 & 0.761 & 0.411 \\
\hline
\end{tabular}

Notes: The table shows the effects of various benefit schemes on the average levels of consumption and the fraction of agents that are at the unconstrained optimum. $b_{j}$ is the level of benefits given in period $j+1$ of an unemployment spell. Hence, $j=0$ corresponds to benefits received during the first quarter, $j=1$ during the second and so on. $b^{*}$ denotes the optimal policy. As discussed in text we have $b_{0}^{*}=1.55 b_{1}^{*}=0.1 b_{\geq 2}^{*}=0.25$. For the remaining columns the level of unemployment benefits in $j$ is reported when $b_{j}>0$.

$\bar{c}^{e}$ denotes average consumption of employed individuals. $\bar{c}_{j}^{u}$ denotes average consumption of unemployed agents where $j=0,1, \geq 2$ denotes the duration in unemployment. With $\geq 2$ I pool together all agents who have been unemployed for at least two quarters. 
Table 3: UI Scheme and Severance Pay

\begin{tabular}{|c|c|c|}
\hline & (1) & $(2)$ \\
\hline 1 & $\mathrm{AK}$ & $\mathrm{AZ}$ \\
\hline 2 & $\mathrm{AR}$ & $\mathrm{CA}$ \\
\hline 3 & $\mathrm{CO}$ & $\mathrm{CT}$ \\
\hline 4 & $\mathrm{DE}$ & $\mathrm{FL}$ \\
\hline 5 & $\mathrm{DC}$ & GA \\
\hline 6 & ID & $\mathrm{HI}$ \\
\hline 7 & IN & IL \\
\hline 8 & IA & KY \\
\hline 9 & $\mathrm{KS}$ & MA \\
\hline 10 & LA & $\mathrm{MO}$ \\
\hline 11 & $\mathrm{ME}$ & NV \\
\hline 12 & $\mathrm{MD}$ & NY \\
\hline 13 & MI & $\mathrm{NC}$ \\
\hline 14 & $\mathrm{MN}$ & OR \\
\hline 15 & MS & RI \\
\hline 16 & $\mathrm{NE}$ & $\mathrm{SC}$ \\
\hline 17 & $\mathrm{NH}$ & $\mathrm{TN}$ \\
\hline 18 & NJ & TX \\
\hline 19 & $\mathrm{OH}$ & VT \\
\hline 20 & $\mathrm{OK}$ & WV \\
\hline 21 & PA & WY \\
\hline 22 & $\mathrm{SD}$ & \\
\hline 23 & $\mathrm{UT}$ & \\
\hline 24 & $\mathrm{VA}$ & \\
\hline 25 & WA & \\
\hline 26 & WI & \\
\hline
\end{tabular}

Notes: The table shows how public employment insurance authorities across US states give out benefits conditional on laid off workers receiving severance compensation from their previous employer. There are 47 states in total ( 3 states are missing). The data were collected by phone interviews with the personnel. Unemployment insurance in states under column (1) is given out as follows: If the applicant has received severance payment from their employer then benefits are reduced by the weekly prorated amount of the payment. Under column (2) benefits are either reduced in the week the severance payment is received or are not take into account altogether. The latter case corresponds to several states (namely California, Hawaii, Illinois, Kentucky, Missouri, New York, Oregon, South Carolina and West Virginia). 


\section{B Online Appendix- Not For Publication}

\section{B.1 Derivations in the Model of Section 3}

I derive the first order conditions from worker's program (2), assuming (for completeness that the worker's constraint may bind). I attach a multiplier $\phi^{1}$ to the worker's constraint and $\phi^{2}$ to the firm's constraint. According to the notation followed in text $\phi^{1}=0, \phi^{2}=\phi$.

The first order conditions from (2) are given by the following equations:

$$
\begin{gathered}
-\frac{1}{c}+\beta \lambda U_{a^{\prime}}\left(a^{\prime}, 0\right)+\beta(1-\lambda) W_{a^{\prime}}\left(a^{\prime}, J^{\prime}\right)+\phi^{1}\left(W_{a^{\prime}}\left(a^{\prime}, J^{\prime}\right)-U_{a^{\prime}}\left(a^{\prime}, 0\right)\right)+\chi=0 \\
\frac{1}{c} \beta(1-\lambda)+\beta(1-\lambda) W_{J^{\prime}}\left(a^{\prime}, J^{\prime}\right)+\phi^{1} W_{J^{\prime}}\left(a^{\prime}, J^{\prime}\right)+\phi^{2}=0 \\
W_{a}(a, J)=\frac{r}{c} \quad \text { and } \quad W_{J}(a, J)=-\frac{1}{c}
\end{gathered}
$$

$W_{x}$ is the partial derivative of $W$ with respect to argument $x$.

Again let $c_{t}^{e}$ denote the consumption of the employed agent in $t$ and $c_{t}^{u}$ the analogous object for the unemployed agent (in the first period of unemployment). With the appropriate substitutions we have:

$$
\begin{gathered}
\left.\frac{1}{c_{t}^{e}}=\beta \lambda r \frac{1}{c_{t+1}^{u}}+\beta(1-\lambda) r \frac{1}{c_{t+1}^{e}}+\phi_{t}^{1} r\left(\frac{1}{c_{t+1}^{e}}-\frac{1}{c_{t+1}^{u}}\right)\right)+\chi_{t} \\
\frac{1}{c_{t}^{e}} \frac{(1-\lambda)}{R}=\beta(1-\lambda) \frac{1}{c_{t+1}^{e}}+\phi_{t}^{1} \frac{1}{c_{t+1}^{e}}-\phi_{t}^{2}
\end{gathered}
$$

Rearranging (using (13) to solve for $\frac{1}{c_{t}^{e}}$ and substituting in (12)) we get:

$$
\frac{R}{(1-\lambda)}\left(\beta(1-\lambda) \frac{1}{c_{t+1}^{e}}+\phi_{t}^{1} \frac{1}{c_{t+1}^{e}}-\phi_{t}^{2}\right)-\beta(1-\lambda) r \frac{1}{c_{t+1}^{e}}-\phi_{t}^{1} r\left(\frac{1}{c_{t+1}^{e}}-\frac{1}{c_{t+1}^{u}}\right)-\chi_{t}=\frac{1}{c_{t+1}^{u}} \beta \lambda r
$$

which can be further rearranged into:

$$
\frac{c_{t+1}^{e}}{c_{t+1}^{u}}=1+\frac{\beta(R-r)}{r\left(\lambda \beta-\phi_{t}^{1}\right)}-\frac{\chi_{t} c_{t+1}^{e}}{r\left(\lambda \beta-\phi_{t}^{1}\right)}+\frac{R}{(1-\lambda)} \frac{1}{r\left(\lambda \beta-\phi_{t}^{1}\right)}\left(\phi_{t}^{1}-\phi_{t}^{2} c_{t+1}^{e}\right)
$$

From (12), (13) and (14) we can obtain the following if we set $\phi_{t}^{1}=0$ and $\phi_{t}^{2}=\phi_{t}$ and make use of $R=\frac{1}{\beta}$ :

$$
\begin{gathered}
\frac{1}{c_{t}^{e}} \beta(1-\lambda)=\beta(1-\lambda) \frac{1}{c_{t+1}^{e}}-\phi_{t} \\
\frac{c_{t+1}^{e}}{c_{t+1}^{u}}=1+\frac{\left(\frac{1}{\beta}-r\right)}{r \lambda}-\frac{\chi_{t} c_{t+1}^{e}}{r \lambda \beta}-\frac{1}{(1-\lambda)} \frac{1}{r \lambda \beta^{2}} \phi_{t} c_{t+1}^{e}
\end{gathered}
$$

which are equations: (7) and (8) in text. The Euler equation follows trivially from (12).

\section{B.2 Analytical Derivations}

I now derive analytically the results of section 3.3. To make the analysis more general I assume first that the interest rate $r$ may exceed 1. I will subsequently use the derivations of this subsection to show additional output from the model.

Under assumptions A.3 (but with $r \geq 1$ ) it is straightforward to establish that consumption 
during unemployment equals $r a_{t}(1-\beta)$. The utility of the unemployed agent is given by:

$$
U(a)=\frac{\log (a)}{1-\beta}+\sum_{j=0}^{\infty} \beta^{j} \log \left(\beta^{j} r^{j}(1-\beta) r\right)
$$

Now consider the employed worker's program. In the initial period the value function is given by $W\left(a_{0}, 0\right)$. Let us first focus on the case where the firm's participation constraint does not bind. The worker must decide on an initial wage $w_{0}$ and a constant wage $\bar{w}$, from period one onwards. Moreover, by the firm's promise keeping constraint, it must be that $w_{0}=y+(y-\bar{w}) \frac{1-\lambda}{R-1+\lambda} \equiv y+(y-\bar{w}) \epsilon$.

As established in text, when the firm's constraint does not bind, consumption remains constant over the life of the match. With initial assets $a_{0}$, and a constant consumption $c^{e}$ it must be that:

$$
\begin{gathered}
a_{1}=r a_{1}+\bar{w}-c^{e} \rightarrow c^{e}=\bar{w}+a_{1}(r-1) \\
a_{1}=r a_{0}+w_{0}-c^{e}=r a_{0}+y+(y-\bar{w}) \epsilon-\bar{w}-(r-1) a_{1} \rightarrow a_{1}=a_{0}+\frac{1}{r}[y+(y-\bar{w}) \epsilon-\bar{w}]
\end{gathered}
$$

Notice that since consumption and the wealth level $\left(a_{1}\right)$ are constant, the worker's value function satisfies: $W\left(a_{0}, 0\right)=W\left(a_{1}, J_{1}\right)$. Lifetime utility also remains constant over time. We can write:

$$
W\left(a_{0}, 0\right)=\max _{\bar{w}} \frac{1}{1-\beta(1-\lambda)}\left(\log \left(a_{1}(r-1)+\bar{w}\right)+\beta \lambda U\left(a_{1}\right)\right)
$$

The first order condition with respect to $\bar{w}$ (and taking into account the expression for $a_{1}$ derived previously) defines the optimum. We have that:

$$
\frac{1}{(r-1) a_{0}+\frac{(y-\bar{w})(1+\epsilon)}{r}+\bar{w}}\left[-\frac{r-1}{r}(1+\epsilon)+1\right]-\frac{\beta \lambda}{(1-\beta) r}(1+\epsilon) \frac{1}{a_{0}+\frac{(y-\bar{w})(1+\epsilon)}{r}}
$$

which can be rewritten as:

$$
\xi_{1}\left[a_{0}+\frac{(y-\bar{w})(1+\epsilon)}{r}\right]=\xi_{2}\left[(r-1) a_{0}+\frac{(y-\bar{w})(1+\epsilon)}{r}+\bar{w}\right]
$$

where $\xi_{1}=1-\frac{r-1}{r}(1+\epsilon)$ and $\xi_{2}=\frac{\beta \lambda}{(1-\beta) r}(1+\epsilon)$. We can derive the optimal wage $\bar{w}$ as:

$$
\bar{w}=\frac{\left[a_{0}+\frac{(1+\epsilon) y}{r}\right]\left(\xi_{1}-\xi_{2}(r-1)\right)}{\xi_{2}+\left(\xi_{1}-\xi_{2}(r-1)\right) \frac{1+\epsilon}{r}}
$$

The participation constraint of the firm is then satisfied if

$$
\frac{\left[a_{0}+\frac{(1+\epsilon) y}{r}\right]\left(\xi_{1}-\xi_{2}(r-1)\right)}{\xi_{2}+\left(\xi_{1}-\xi_{2}(r-1)\right) \frac{1+\epsilon}{r}} \leq y
$$

To prove Result 1 in text let $r=1$. In this case we have that $\xi_{1}=1$ and $\xi_{2}=\frac{\beta \lambda}{(1-\beta)}(1+\epsilon)=$ $\frac{\beta \lambda}{(1-\beta)} \frac{1}{(1-\beta+\beta \lambda)}$. (17) and (18) become: $\bar{w}=a_{0}(1-\beta)+\frac{y(1-\beta)}{1-\beta+\beta \lambda}$ and $a_{0}(1-\beta) \leq y \frac{\beta \lambda}{1+\beta \lambda-\beta}$ respectively.

The worker's participation constraint. To complete these derivations, let me show now that the participation contraint of the worker is slack in the unconstrained optimum. I assume for simplicity that $r=1$. I need to show that:

$$
W\left(a_{1}, J_{1}\right)>U\left(a_{1}, 0\right) \quad \text { or } \quad \frac{1}{1-\beta(1-\lambda)}\left(\log (\bar{w})+\beta \lambda U\left(a_{1}, 0\right)\right)>U\left(a_{1}, 0\right)
$$


Making use of the above formulas (19) becomes:

$$
\frac{\log (\bar{w})}{1-\beta}>\frac{\log \left(a_{0} \beta \lambda(1+\epsilon)+y \beta \lambda(1+\epsilon)^{2}\right)}{1-\beta}+\frac{\log (1-\beta)}{1-\beta}+\beta \log (\beta) \frac{1}{(1-\beta)^{2}}
$$

(20) then gives:

$$
\frac{\log (1-\beta)}{1-\beta}>\frac{\log (\beta \lambda(1+\epsilon))}{1-\beta}+\frac{\log (1-\beta)}{1-\beta}+\beta \log (\beta) \frac{1}{(1-\beta)^{2}}
$$

or

$$
0>\log \left(\frac{\lambda}{1-\beta+\beta \lambda}\right)+\log (\beta) \frac{1}{(1-\beta)}
$$

The second term on the LHS in (21) is negative as $\beta$ is less than one. Moreover, the leading term is also negative if $\lambda$ is less than one. Therefore the inequality in (21) holds, proving that the allocation does not violate the worker's participation constraint.

In the case where $a_{0}(1-\beta)>y \frac{\beta \lambda}{1+\beta \lambda-\beta}$ (the firm's constraint is binding) A.2. guarantees that the worker's constraint is not binding.

\section{B.2.1 Effect of interest rates}

Now consider the effect of the interest rate $r$ on wages, the level of wealth above which the firm's constraint binds and the worker's participation constraint. We can show the following: i) when $r$ increases the initial wage $w_{0}$ is larger. ii) the participation constraint of the firm now binds at a higher wealth level $a_{0}$ than before. iii) the participation constraint of the worker will not bind for any $r<\frac{1}{\beta}$.

i) and ii) can be explicitly shown using equations (17) and (18). For the sake of brevity, I only show that the worker's participation constraint is slack so that indeed (17) represents an unconstrained optimum. We need to show that:

$$
\frac{1}{1-\beta(1-\lambda)}\left[\log \left(c^{e}\right)+\beta \lambda U\left(a_{1}, 0\right)\right]>U\left(a_{1}, 0\right) \rightarrow \log \left(c^{e}\right)>(1-\beta) U\left(a_{1}, 0\right)
$$

where $c^{e}=a_{1}(r-1)+\bar{w}$. For simplicity, let us write $\bar{w}=\frac{\left[a_{0}+\frac{(1+\epsilon) y}{r}\right] \widetilde{\omega}_{1}}{\widetilde{\omega}_{2}}$. Then $a_{1}=1-\frac{\left[a_{0}+\frac{(1+\epsilon) y}{r}\right] \widetilde{\omega}_{1}}{\widetilde{\omega}_{2}}$. From this condition we can derive the participation constraint as follows:

$\log \left((r-1)\left[1-\frac{\left[a_{0}+\frac{(1+\epsilon) y}{r}\right] \widetilde{\omega}_{1}}{\widetilde{\omega}_{2}}\right]+\frac{\left[a_{0}+\frac{(1+\epsilon) y}{r}\right] \widetilde{\omega}_{1}}{\widetilde{\omega}_{2}}\right) \geq \log \left(1-\frac{\left[a_{0}+\frac{(1+\epsilon) y}{r}\right] \widetilde{\omega}_{1}}{\widetilde{\omega}_{2}}\right)+\sum_{j=0}^{\infty} \beta^{j} \log \left(\beta^{j} r^{j}(1-\beta) r\right)$

This can be rearranged into:

$$
\log \left[(r-1)+\frac{\widetilde{\omega}_{1}}{\widetilde{\omega}_{2}-\widetilde{\omega}_{1}}\right]-\log (1-\beta) r-\frac{\beta}{1-\beta} \log (r \beta) \geq 0
$$

This equation cannot be simplified ( $\widetilde{\omega}_{1}$ and $\widetilde{\omega}_{2}$ are nonlinear in $\beta$ and $r$ ). However, we can show that when $r=\frac{1}{\beta}$ condition (22) becomes $\log (r-1)-\log (r-1) \geq 0$ and we can also show (through taking derivatives) that when $r<\frac{1}{\beta}$ and $\beta<1$ the LHS is monotonically decreasing in $r$. In other words, the worker's constraint is always satisfied under A.1. For brevity the derivations are omitted.

\section{B.2.2 Non-Divergent Savings....}

Let me now assume that $r=\frac{1}{\beta}$. As is well known, when individuals face idiosyncratic income risks and markets are incomplete, assuming that the interest rate is equal to the inverse of the discount 
factor leads household savings to diverge to infinity (see for example Aiyagari (1994)). In the current setup when we assume that wage contracts are flat this can be illustrated as follows: Assume that the worker earns $y$ every period. The euler equation is given by:

$$
\frac{1}{c_{t}^{e}}=\beta r(1-\lambda) \frac{1}{c_{t+1}^{e}}+\beta r \lambda \frac{1}{c_{t+1}^{u}}
$$

where (as previously) $c_{t+1}^{u}=(1-\beta) r a_{t+1}$ denotes consumption during unemployment.

Assume that there is a stationary (finite) level of wealth which, once reached, the worker does not further accumulate wealth. Let this level be $\widetilde{a}$. At $a_{t}=a_{t+1}=\widetilde{a}$ it must be that $c_{t}^{e}=c_{t+1}^{e}$ (consumption remains constant during employment). Then, from the Euler equation we get: $c_{t+1}^{e}=$ $c_{t+1}^{u}$ if $\beta r=1$. Now take the budget constraint of the worker. We have that:

$$
a_{t+2}=r a_{t+1}+y-c_{t+1}^{e}=r a_{t+1}+y-(1-\beta) r a_{t+1}=a_{t+1}+y
$$

This implies that the law of motion of assets evaluated at $\widetilde{a}$ is a linear difference equation with a unit root and a constant forcing term equal to $y>0$. This equation obviously diverges to infinity and contradicts that there exists $\widetilde{a}<\infty$ which is a stationary wealth level.

Divergent savings do not obtain when we assume the optimal contract. If we have $r=\frac{1}{\beta}$ then $\xi_{1}-\xi_{2}(r-1)=0^{31}$. From equation (17) we get that $\bar{w}=0$. The worker borrows from the firm in the first period the entire present value of output. Consumption in every period is $c^{e}=(r-1) a_{1}=$ $(1-\beta) r a_{1}=c^{u}{ }^{32}$ The worker effectively faces no idiosyncratic risks from period 1 onwards since income in employment is zero (as it is in unemployment under A.3) and consumption does not drop if the worker becomes unemployed. The firm's constraint is clearly not binding since after the initial loan the firm receives $y$ in every period. The worker's constraint is also not violated; given that the worker receives an income equal to zero in every period, she becomes indifferent between remaining employed and becoming unemployed. The constraint is satisfied with equality. This was proved explicitly in the previous paragraph (e.g. equation (22)).

The result highlighted in this subsection is clearly an isolated case. It relies strongly on the assumption that income during unemployment is constant and that unemployment is an absorbing state. These are clearly very restrictive assumptions.

\section{B.3 Accounting for the Worker's Participation Constraint}

In the paper I omitted the explicit treatment of the worker's constraint. Under A.1. this constraint did not bind in any of the models I solved. Though there is no analytical proof, one should expect $W\left(a^{\prime}, J^{\prime}\right) \geq U\left(a^{\prime}, 0\right)$ to bind in equilibrium, when we have that $R=r$ (see Oikonomou (2010)). As I demonstrate in this section, in this case the optimal allocation gives a decreasing profile $c_{t}^{e}$. When consumption during employment reaches a very low level the worker effectively becomes indifferent between employment and unemployment. ${ }^{33}$ In this setup, if we assume full commitment (and hence ignore the constraint $W\left(a^{\prime}, J^{\prime}\right) \geq U\left(a^{\prime}, 0\right)$ altogether), we would obtain $\lim _{t \rightarrow \infty} c_{t}^{e}=0$. The worker's participation constraint does not allow the marginal utility of consumption to diverge to infinity.

Let me for simplicity use the previous derivations ((12), (13) and (14)) imposing that $\phi_{t}^{2}=0$ (the firm's constraint doesn't bind). Since in a match with positive surplus (e.g. A.2) we will not have that the firm's and the worker's participation constraint bind simultaneously we can study separately

\footnotetext{
${ }^{31} \xi_{1}-\xi_{2}(r-1)=1-\frac{r-1}{r}(1+\epsilon)-(r-1) \frac{\beta \lambda}{(1-\beta) r}(1+\epsilon)=1-\frac{r-1}{r} \frac{1}{1-\beta+\beta \lambda}\left(1+\frac{\beta \lambda}{(1-\beta)}\right)=1-\frac{r-1}{r(1-\beta)}=1-\frac{r-1}{r-1}=0$

${ }^{32}$ As established in text, when the worker and the firm discount the future at equal rates, the worker is perfectly insured against unemployment (her consumption does not drop in the first period).

${ }^{33}$ When $R=\frac{1}{\beta}$ the worker's consumption is constant. If the constraint of the worker binds, it must bind from the first period. This was shown analytically in the example in subsection B.1.2. If we maintain that $W\left(a_{0}, 0\right)>U\left(a_{0}, 0\right)$ it must be that the worker desires to borrow a large amount from the firm so that $W\left(a_{1}, J_{1}\right)=U\left(a_{1}, 0\right)$. However, as we have seen, because $r<R$ the worker wants to borrow relatively little. The value of $J_{1}$ will never be so high so that the worker wants to quit and become unemployed.
} 
the case where $\phi_{t}^{1}>0$. I can write the optimality conditions as follows:

$$
\begin{gathered}
\left.\frac{1}{c_{t}^{e}}=\beta \lambda r \frac{1}{c_{t+1}^{u}}+\beta(1-\lambda) r \frac{1}{c_{t+1}^{e}}+\phi_{t}^{1} r\left(\frac{1}{c_{t+1}^{e}}-\frac{1}{c_{t+1}^{u}}\right)\right)+\chi_{t} \\
\frac{1}{c_{t}^{e}} \frac{(1-\lambda)}{R}=\beta(1-\lambda) \frac{1}{c_{t+1}^{e}}+\phi_{t}^{1} \frac{1}{c_{t+1}^{e}} \\
\frac{c_{t+1}^{e}}{c_{t+1}^{u}}=1+\frac{\beta(R-r)}{r\left(\lambda \beta-\phi_{t}^{1}\right)}-\omega+\frac{R}{(1-\lambda)} \frac{1}{r\left(\lambda \beta-\phi_{t}^{1}\right)} \phi_{t}^{1}
\end{gathered}
$$

The following results emerge from these conditions:

1. Under $\phi_{t}^{1}>0$ the standard Euler equation does not hold as an equality.

(23) represents a modified Euler equation (see LTW). Since assets $a_{t+1}$ impact the constraint $W\left(a_{t+1}, J_{t+1}\right) \geq U\left(a_{t+1}, 0\right)$, the Euler equation needs to be augmented to include the term $\left.\phi_{t}^{1} r\left(\frac{1}{c_{t+1}^{e}}-\frac{1}{c_{t+1}^{u}}\right)\right)$. The impact of this term is the following: assume that $c_{t+1}^{e}>c_{t+1}^{u}$. Then,

$$
\frac{1}{c_{t}^{e}}<\beta \lambda r \frac{1}{c_{t+1}^{u}}+\beta(1-\lambda) r \frac{1}{c_{t+1}^{e}}+\chi_{t}
$$

The cost of an extra unit of savings is less than the future benefit, the agent is savings constrained. ${ }^{34}$

\section{Consumption increases during employment.}

This can be seen from (24). As discussed previously, the term $\frac{1}{c_{t}^{e}}$ represents the relative weight of the firm and the worker in the optimal allocation (see the next subsection for the derivation). When the worker's constraint binds her relative weight must increase over time so that $\frac{1}{c_{t}^{e}}$ drops.

3. Consumption may drop during unemployment more when the worker's constraint binds.

This can be seen from (25). When $\phi_{t}^{1}=0$ (and $\chi_{t}=0$ ) we have that $\frac{c_{t+1}^{e}}{c_{t+1}^{u}}=1+\frac{\beta(R-r)}{r(\lambda \beta)}$. This is smaller than the value of $(25)$ when $\phi_{t}^{1}>0$ (unless $\left.\phi_{t}^{1}>\lambda \beta\right) .{ }^{35}$ Note that even in the latter case, the ratio $\frac{c_{t+1}^{e}}{c_{t+1}^{u}}$ may be less than one.

Unfortunately, it is difficult to fully characterize the optimal wage schedule in the case where the worker's constraint binds. We can show formally the following: i) under A.1, if the constraint binds in a given period $T$ then it binds in $t=1,2,3, \ldots$ (in other words it binds forever). ${ }^{36}$. ii)if the worker's constraint binds then it must be that $w_{0}>y .{ }^{37}$ However, it is impossible to pin down wages in any other period beyond period 0 because it is also impossible to pin down the behavior of assets

\footnotetext{
${ }^{34}$ We can interpret this implication based on the discussion of the analogous result in Ligon et al (2000). In the case where the worker's constraint is binding, the worker needs to be compensated with a higher consumption level in $t+1$. The higher consumption can be financed either through higher wealth or through a larger wage in $t+1$. In the case where $c_{t+1}^{e}>c_{t+1}^{u}$, increasing consumption with assets, tightens the participation constraint. In this case (as discussed previously) the worker is savings constrained (she wants to save more but is unable). The opposite holds when $c_{t+1}^{e}<c_{t+1}^{u}$.

${ }^{35} \mathrm{~A}$ first order approximation to $(25)$ around $\phi_{t}^{1}=0$ always yields that consumption drops more during unemployment if the constraint binds.

${ }^{36} \mathrm{~A}$ formal proof of this claim can be made along the lines of the proofs of propositions 2 and 3 . In particular, the proof of proposition 3 can be repeated to show that the constraint binds for $t=1,2, \ldots, T$. A similar argument to the one developed in the appendix to prove proposition 4 , can be invoked to show that it must be that $T=\infty$. For brevity the proof is omitted.

37 We have that $W\left(a_{1}, J_{1}\right)=U\left(a_{1}, 0\right)$ and from A.2 $W\left(a_{1}, 0\right)>U\left(a_{1}, 0\right)$ (if $J_{1}<0$ the firm's constraint is violated and clearly then $\left.U\left(a_{1}, 0\right)<W\left(a_{1}, 0\right)<W\left(a_{1}, J_{1}\right)\right)$. Hence, it must be that $J_{1}>0$, which implies that wages initially exceed $y$.
} 
over time. ${ }^{38}$

A final comment is in order: Notice that when the worker's constraint is the one which is crucial in equilibrium, a completely different set of results could arise in terms of the optimal benefit policies. The government is now concerned to drive the allocation away from the point where the worker is indifferent between employment and unemployment. Maximizing welfare may require to have as few individuals as possible at the participation constraint. This can be accomplished through backloading unemployment benefits opposite to the result which I derived in the paper (see for example Oikonomou (2010, Ch. 2). The implications of the limited commitment model for UI policy therefore hinge on which of the two participation constraints is most relevant. Nevertheless, having a model where consumption drops over time for employed individuals seems counterfactual. I therefore chose to work with the case $R=\frac{1}{\beta}>r$ following the literature on optimal contracts.

\section{B.4 A Recursive Contract Representation}

In this subsection I derive the worker's program using a Lagrangian formulation. I show that the marginal utility of consumption of the worker, represents the relative weights of the firm and the worker in the Pareto optimal allocation. In text I had used this result (which is standard in the literature) to interpret the law of motion of $c_{t}^{e}$ over time (e.g. equation (7) in text). Let $\kappa_{w}$ represent an initial weight attached to the worker's wellbeing, and $\kappa_{f}$ the analogous weight attached to the firm. The problem which needs to be resolved is the following:

$$
\begin{gathered}
\max \sum_{0}^{\infty} \beta^{t}(1-\lambda)^{t}\left[\kappa_{w}\left(\log \left(-a_{t+1}+r a_{t}+w_{t}-\tau\right)+\beta \lambda U\left(a_{t+1}, 0\right)\right)+\kappa_{f}\left(y-w_{t}\right)\right] \\
\text { st } \sum_{j=0}^{\infty} \beta^{j}(1-\lambda)^{j}\left[y-w_{t+j}\right] \geq 0 \\
\sum_{j=0}^{\infty} \beta^{j}(1-\lambda)^{j}\left[\log \left(-a_{t+1+j}+r a_{t+j}+w_{t+j}-\tau\right)+\beta \lambda U\left(a_{t+j+1}, 0\right)\right] \geq U\left(a_{t}, 0\right) \\
a_{t+1} \geq 0
\end{gathered}
$$

If (for simplicity) we leave out the worker's participation constraint, we can write:

$$
\begin{gathered}
L=\sum_{0}^{\infty} \beta^{t}(1-\lambda)^{t}\left[\kappa_{w}\left(\log \left(-a_{t+1}+r a_{t}+w_{t}-\tau\right)+\beta \lambda U\left(a_{t+1}, 0\right)\right)+\kappa_{f}\left(y-w_{t}\right)\right] \\
\left.+\phi_{t}\left(\sum_{j=0}^{\infty} \beta^{j}(1-\lambda)^{j}\left[y-w_{t+j}\right]-0\right)-\chi_{t}\left(a_{t+1}-0\right)\right]
\end{gathered}
$$

which can be rearranged into:

$L=\sum_{0}^{\infty} \beta^{t}(1-\lambda)^{t}\left[\kappa_{w}\left(\log \left(-a_{t+1}+r a_{t}+w_{t}-\tau\right)+\beta \lambda U\left(a_{t+1}, 0\right)\right)+\left(\kappa_{f}+\bar{\phi}_{t}\right)\left(y-w_{t}\right)-\chi_{t}\left(a_{t+1}-0\right)\right]$

${ }^{38}$ To see this note that the following sequence of equalities need to hold (since the constraint always binds):

$$
\sum_{j=0}^{\infty} \beta^{j}(1-\lambda)^{j}\left[\log \left(c_{t+j}^{e}\right)+\beta \lambda U\left(a_{t+j+1}, 0\right)\right]=U\left(a_{t}, 0\right)
$$

Moreover, since $c_{t}^{e}$ increases over time under a binding participation constraint for the worker we can rule out the case where assets remain constant over time. However, we cannot rule out paths where $a_{t}$ increases over time nor paths where wealth drops (at least for a few periods). Moreover, even if we know that $a_{t}$ increases monotonically, this still does not help us identify the path of wages (higher consumption could be financed through higher wealth so that wages could decrease or increase and so on). 
where $\bar{\phi}_{t}=\bar{\phi}_{t-1}+\phi_{t}$ and $\bar{\phi}_{t-1}=0$. The first order condition with respect to $w_{t}$ gives: $\frac{\kappa_{w}}{c_{t}^{e}}=\kappa_{f}+\bar{\phi}_{t}$ which gives the relative weight of the firm and the worker as: $\frac{\kappa_{f}+\bar{\phi}_{t}}{\kappa_{w}}=\frac{1}{c_{t}^{e}}$. This was discussed in section 3.1.1 of the paper. Standard results imply that the above program can be represented recursively, applying the methodology of Marcet and Marimon (2002). For the sake of brevity I omit the Bellman equation.

\section{B.5 Numerical Algorithm}

In order to solve the model I utilize the standard value function iteration approach. However, for simplicity I cast the program in its dual form, considering an optimal contract that maximizes the firm's profit subject to delivering a given level of utility to the worker. This representation is standard in the literature and as discussed, it is equivalent to the representation followed in the main text. The program may be written as follows:

$$
\Pi(W, a)=\max _{a^{\prime}, w, W^{\prime}} y-w+\frac{1-\lambda}{R} \Pi\left(W^{\prime}, a^{\prime}\right)
$$

subject to:

$$
\begin{gathered}
W \leq \log \left(-a^{\prime}+w-\tau+a r\right)+\beta\left((1-\lambda) W^{\prime}+\lambda U\left(a^{\prime}, 0\right)\right) \\
W^{\prime} \geq U\left(a^{\prime}, 0\right) \quad \text { and } \Pi\left(W^{\prime}, a^{\prime}\right) \geq 0
\end{gathered}
$$

where $\Pi(W, a)$ is the profit function of the firm, given a level of promised utility $W$ and given a wealth level $a$. Moreover, equation (27) is the promise keeping constraint stating that $W$ must equal the instantaneous payoff $\log (c)$ (where $c=-a^{\prime}+w-\tau+a r$ ) plus the continuation value $\left.\beta\left[(1-\lambda) W^{\prime}+\lambda U\left(a^{\prime}, 0\right)\right)\right]$. The participation constraints are $W^{\prime} \geq U\left(a^{\prime}, 0\right)$ and $\Pi\left(W^{\prime}, a^{\prime}\right) \geq 0$.

In order to reduce the number of variables (control and states) I make use of the following properties: First, I use the promise keeping constraint (which holds as an equality) to express wages as:

$$
w=u^{-1}\left(W-\beta\left[(1-\lambda) W^{\prime} \lambda U\left(a^{\prime}, 0\right)\right]\right)+a^{\prime}+\tau-r a \equiv e^{\left.W-\beta\left[(1-\lambda) W^{\prime} \lambda U\left(a^{\prime}, 0\right)\right]\right)}+a^{\prime}+\tau-r a
$$

This allows me to write:

$$
\Pi(W, a)=\max _{a^{\prime}, W^{\prime}} y-e^{W-\beta\left[(1-\lambda) W^{\prime} \lambda U\left(a^{\prime}, 0\right)\right]}-a^{\prime}-\tau+r a+\frac{1-\lambda}{R} \Pi\left(W^{\prime}, a^{\prime}\right)
$$

Second, from (29) it is straightforward to show that the firm's value function is linear homogeneous in wealth. ${ }^{39}$ I can solve the functional equation:

$$
\Pi(W, 0)=\max y-u^{-1}\left(W-\beta\left[(1-\lambda) W^{\prime}+\lambda U\left(a^{\prime}, 0\right)\right]\right)-a^{\prime}-\tau+\frac{1-\lambda}{R}\left[\Pi\left(W^{\prime}, 0\right)+a^{\prime} r\right]
$$

to recover the policy rules. Equivalently, the participation constraints are $W^{\prime} \geq U\left(a^{\prime}, 0\right)$ and $\Pi\left(W^{\prime}, 0\right)+r a^{\prime} \geq 0$.

Equation (30) can be solved utilizing a standard value function iteration approach. Moreover, it is efficient to make use of the property that, when the participation constraints are slack, the optimal contract sets $W^{\prime}=W$. The algorithm proceeds as follows:

- Given the value functions $U(a, j)$ for unemployed workers, take a grid of values $\mathcal{W}=\left\{W_{1}, W_{2}, \ldots, W_{n}\right\}$ for promised utlity. Solve the value function (30) on this grid, using cubic spline interpolation to evalute the profit and utility functions on values which fall outside the grid. Recover the optimal policies $W^{\prime}=g_{W}(W)$ and $a^{\prime}=g_{a}(W)$. In particular, start from an initial guess $\Pi_{0}$ and solve the firm's program.

\footnotetext{
${ }^{39}$ The current wealth level $a$ does not affect the participation constraints.
} 
1. Solve first the unconstrained allocation:

$$
\Pi(W, 0)=\max _{a^{\prime} \geq 0} y-u^{-1}\left(W-\beta\left[(1-\lambda) W+\lambda U\left(a^{\prime}, 0\right)\right]\right)-a^{\prime}-\tau+\frac{1-\lambda}{R}\left[\Pi_{0}(W, 0)+a^{\prime} r\right]
$$

i.e. imposing from the outset that the participation constraints are slack and that $W^{\prime}=W$. Then, verify that indeed $W \geq U\left(a^{\prime}, 0\right)$ and $\Pi_{0}(W, 0)+r a^{\prime} \geq 0$. If either of these conditions is violated, then solve the costrained program.

2. For example, if $\Pi(W, 0)+r a^{\prime}<0$, then we know that $W^{\prime} \neq W$ and also that $\Pi_{0}\left(W^{\prime}, 0\right)+$ $r a^{\prime}=0$. We need to solve for the value of $W^{\prime}$ which satisfies the participation contraint with equaility. Note that assets are easy to recover from $r a^{\prime}=-\Pi_{0}\left(W^{\prime}, 0\right)$. This is essentially a problem with one control variable.

3. On the other hand, if the worker's constraint is binding then we have $W^{\prime}=U\left(a^{\prime}, 0\right)$ at the optimum. We then need to find the value of $a^{\prime}$ that maximizes the firm's profits. Again this requires to choose the level of one control variable; hence, it is a simple problem to solve.

From the solution of the Bellman equation we obtain a new function $\Pi_{1}(W, 0)$. If $\Pi_{1}(W, 0)$ and $\Pi_{0}(W, 0)$ are close we have found the convergent solution. If not we iterate until convergence.

- Given the optimal policies $W^{\prime}=g_{W}(W)$ and $a^{\prime}=g_{a}(W)$, we need to recover the level of expected promised utility consistent with the zero profit condition. This is a function $\widetilde{W}(a)$ which solves the nonlinear system of equations $\Pi(\widetilde{W}(a), 0)+r a=0$. Given $\widetilde{W}(a)$ we update the value functions for unemployed workers. We therefore need to solve:

$$
\left.U_{1}(a, j)=\max _{c, a^{\prime} \geq 0, s} \log (c)-v(s)+\beta \gamma(s) \widetilde{W}\left(a^{\prime}\right)+\beta(1-\gamma(s)) U_{0}\left(a^{\prime}, j+1\right)\right)
$$

subject to

$$
a^{\prime}=r a+b_{j}-c
$$

for every $j$. With the updated values $U_{1}(a, j)$ we solve again the firm's problem, obtain a new utility $\widetilde{\widetilde{W}}(a)$ and solve again the program of the unemployed agents.

- Iterate on the above steps until $U(a, j)$ has converged.

The above algorithm focuses on the solution to the Bellman equation. For the sake of brevity I omitted the description the computation of the steady state distribution.

\section{B.6 The first order conditions from the profit maximization problem}

I now show that through maximizing the firm's profits we can derive the same first order conditions characterizing the optimal allocation as from the worker's program described in text. Let (following the notation of the previous subsection) $\Pi(W, a)$ be the profit function where $W$ is promised utility to the worker. We have:

$$
\begin{gathered}
\Pi(W, a)=\max _{a^{\prime}, w, W^{\prime}} y-w+ \\
+\frac{1-\lambda}{R} \Pi\left(W^{\prime}, a^{\prime}\right)-\widetilde{\psi}\left(W-\log \left(-a^{\prime}+w-\tau+a r\right)-\beta\left((1-\lambda) W^{\prime}+\lambda U\left(a^{\prime}, 0\right)\right)\right. \\
+\bar{\phi}^{1}\left(W^{\prime}-U\left(a^{\prime}, 0\right)\right)+\bar{\phi}^{2}\left(\Pi\left(W^{\prime}, a^{\prime}\right)-0\right)+\bar{\chi}\left(a^{\prime}-0\right)
\end{gathered}
$$


where primes as usual denote next period variables. The first order conditions (making use of time subscripts for convenience give the following:

$$
\begin{gathered}
\Pi_{W}\left(W_{t}, a_{t}\right)=-\widetilde{\psi}_{t} \\
\Pi_{a}\left(W_{t}, a_{t}\right)=\widetilde{\psi}_{t} \frac{r}{c_{t}^{e}} \\
-1+\widetilde{\psi}_{t} \frac{1}{c_{t}^{e}}=0 \\
\frac{1-\lambda}{R} \Pi_{W}\left(W_{t+1}, a_{t+1}\right)+\widetilde{\psi}_{t} \beta\left((1-\lambda)+\bar{\phi}_{t}^{1}+\bar{\phi}_{t}^{2} \Pi_{W}\left(W_{t+1}, a_{t+1}\right)=0\right. \\
\frac{1-\lambda}{R} \Pi_{a}\left(W_{t+1}, a_{t+1}\right)-\widetilde{\psi}_{t} \frac{1}{c_{t}^{e}}+\widetilde{\psi}_{t} \beta \lambda \frac{r}{c_{t+1}^{u}}-\bar{\phi}_{t}^{1} \frac{r}{c_{t+1}^{u}}+\bar{\phi}_{t}^{2} \Pi_{a}\left(W_{t+1}, a_{t+1}\right)+\bar{\chi}_{t}=0
\end{gathered}
$$

From the above we get: $\widetilde{\psi}_{t}=c_{t}^{e}$ and $\Pi_{a}\left(W_{t}, a_{t}\right)=r$ (linearity in wealth). The fourth equation above then gives:

$$
\begin{gathered}
-\beta(1-\lambda) c_{t+1}^{e}+c_{t}^{e} \beta\left((1-\lambda)+\bar{\phi}_{t}^{1}-\bar{\phi}_{t}^{2} c_{t+1}^{e}=0 \rightarrow\right. \\
\beta(1-\lambda) \frac{1}{c_{t}^{e}}=\beta\left((1-\lambda) \frac{1}{c_{t+1}^{e}}+\bar{\phi}_{t}^{1} \frac{1}{c_{t}^{e} c_{t+1}^{e}}-\bar{\phi}_{t}^{2} \frac{1}{c_{t}^{e}}\right.
\end{gathered}
$$

Define now $\bar{\phi}_{t}^{2} \frac{1}{c_{t}^{e}} \equiv \phi_{t}^{2}$ and $\bar{\phi}_{t}^{1} \frac{1}{c_{t}^{e}} \equiv \phi_{t}^{1}$. We then have:

$$
\beta(1-\lambda) \frac{1}{c_{t}^{e}}=\beta\left((1-\lambda) \frac{1}{c_{t+1}^{e}}+\phi_{t}^{1} \frac{1}{c_{t+1}^{e}}-\phi_{t}^{2}\right.
$$

Indeed this is the first condition characterizing the optimum in the worker's program. Moreover, from the above we have:

$$
\begin{gathered}
\frac{1-\lambda}{R} r \frac{1}{c_{t}^{e}}-\frac{1}{c_{t}^{e}}+\beta \lambda \frac{r}{c_{t+1}^{u}}-\bar{\phi}_{t}^{1} \frac{1}{c_{t}^{e}} \frac{r}{c_{t+1}^{u}}+\bar{\phi}_{t}^{2} \frac{1}{c_{t}^{e}} r+\bar{\chi}_{t} \frac{1}{c_{t}^{e}}=0 \rightarrow \\
1-\lambda \beta r \frac{1}{c_{t}^{e}}-\frac{1}{c_{t}^{e}}+\chi_{t}+\beta \lambda \frac{r}{c_{t+1}^{u}}-\phi_{t+1}^{1} \frac{r}{c_{t+1}^{u}}+\phi_{t}^{2} r=0
\end{gathered}
$$

where $\bar{\chi}_{t} \frac{1}{c_{t}^{e}}=\chi_{t}$. Substituting out $1-\lambda \beta r \frac{1}{c_{t}^{e}}=r \beta\left((1-\lambda) \frac{1}{c_{t+1}^{e}}+r \phi_{t}^{1} \frac{1}{c_{t+1}^{e}}-r \phi_{t}^{2}\right.$ we get:

$$
r \beta\left((1-\lambda) \frac{1}{c_{t+1}^{e}}+r \phi^{1}{ }_{t} \frac{1}{c_{t+1}^{e}}-\frac{1}{c_{t}^{e}}+\beta \lambda \frac{r}{c_{t+1}^{u}}-\phi_{t}^{1} \frac{r}{c_{t+1}^{u}}+\chi_{t}=0\right.
$$

Rearranging:

$$
\frac{1}{c_{t}^{e}}=r \beta\left((1-\lambda) \frac{1}{c_{t+1}^{e}}+\beta \lambda \frac{r}{c_{t+1}^{u}}+\phi_{t}^{1} r\left(\frac{1}{c_{t+1}^{e}}-\frac{1}{c_{t+1}^{u}}\right)+\chi_{t}\right.
$$

which is the Euler equation derived from the worker's program. As discussed in the appendix of the paper through combining (31) and (32) we can obtain the expression for $\frac{c_{t+1}^{e}}{c_{t+1}^{u}}$ derived in the text and the appendix.

\section{B.7 Severance Payments}

In this subsection I prove the claim made in text that the frontloaded wage profile can be decentralized as a constant wage + severance payment scheme. I first show that this holds exactly when I assume that $R=r$, using the Bellman equation of the worker's program. Then, I show that when $R=\frac{1}{\beta} \mathrm{I}$ need to bring additional features to the model, in order to get the result. In both cases the frontloaded 
wage profile can be interpreted as firm insurance through severance payments.

Let severance payment be denoted by $\xi$. Assume for the moment that the participation constraints for the firm and the worker can be ignored. The optimal allocation then solves the following functional equation:

$$
\begin{aligned}
W(a, J) & =\max _{a^{\prime}, J^{\prime}, \xi} \log \left(-a^{\prime}+r a-\tau-J+y+\frac{1-\lambda}{R} J^{\prime}-\frac{\lambda \xi}{R}\right) \\
& +\beta\left(\lambda U\left(a^{\prime}+\frac{\xi}{R}, 0\right)+(1-\lambda) W\left(a^{\prime}, J^{\prime}\right)\right)
\end{aligned}
$$

Subject to the constraint set:

$$
a^{\prime} \geq \bar{a} \quad a^{\prime}+\frac{\xi}{R} \geq \bar{a}
$$

40 Note that in (33) the promise keeping constraint (which holds as an equality) is given by: $J=$ $y-w+\frac{1-\lambda}{R} J^{\prime}-\frac{\lambda}{R} \xi$. In the event of a separation the firm pays to the worker income equal to $\xi$. Moreover, note that after replacing consumption with the promise keeping and budget constraints in (33), what remains in the constraint set is the boundary conditions $a^{\prime} \geq \bar{a}$ and $a^{\prime}+\frac{\xi}{R} \geq \bar{a}$. The latter requires total assets in the event of separation to not violate the borrowing limit.

To show that program (33) is the same as program (2) consider the following Ricardian equivalence argument: Increase wealth for the worker by $\frac{\xi}{R}$ today and let the new level of assets be $\tilde{a}^{\prime}=a^{\prime}+\frac{\xi}{R}$. Also increase the continuation (promised) utility $J^{\prime}$ by $\xi$ and define $\tilde{J}^{\prime}=J^{\prime}+\xi$. Then clearly $W\left(\tilde{a}^{\prime}, \tilde{J}^{\prime}\right)=W\left(a^{\prime}, J^{\prime}\right)$ since the amount of resources available to finance consumption for the worker next period is unchanged. Thus a program that sets $\xi=0$ and uses next period's wealth as a single control variable as in equation (2) is payoff equivalent to one where both investment in wealth and severance payments are allowed. ${ }^{41}$

The above derivations hold exactly in the case $R=r .{ }^{42}$ However, when $R>r$ they do not. To see what the issue is note that in the case of severance payments the worker does not borrow from the firm rather she buys a state contingent asset which pays a unit of income in unemployment. These two options cannot be equivalent for two reasons: First, the state contingent asset could yield complete coverage against unemployment risks (which was not the case with $R>r$ ) and second, under severance payments the worker saves with the firm and with return dominance $(R>r)$ the constraint set is not the same since the higher return $R$ creates wealth intertemporally.

\section{B.7.1 Wages and severance payments when $R=\frac{1}{\beta}$}

I now illustrate that the frontloaded wage profile in the case $R=\frac{1}{\beta}$ may still be interpreted as a severance payment if I let the worker choose $\xi$ under two additional assumptions: 1) the firm makes positive profits from the contract (when optimal $\xi>0$ ) and 2) the worker does not internalize

\footnotetext{
40 Note that it is important to divide the severance pay by $R$. This holds because given that the net interest on savings maybe positive $r-1>0$ the next period's asset for the worker is $a^{\prime} r$ after the return is realized. Therefore, if we increase wages by $\frac{\xi}{R}$ to increase savings, the worker will receive $r \frac{\xi}{R}$. This equals $\xi$ (i.e. the severance pay) when $r=R$.

Moreover, we need to impose both that $a^{\prime} \geq \bar{a}$ and $a^{\prime}+\frac{\xi}{R} \geq \bar{a}$ so that wealth in employment and unemployment does not violate the constraint.

${ }^{41}$ Under limited commitment matters may seem more complicated because of the presence of the sustainability restrictions. A crucial assumption is that that severance are fully enforceable, and also that the worker's outside option is such that she always gets $\xi$ when the match is destroyed. (i.e $U\left(a^{\prime}+\frac{\xi}{R}, 0\right)$ ), as opposed to $U\left(a^{\prime}, 0\right)$. This is obviously relevant in cases where the worker's constraint may bind. For instance, consider a comparison between $\tilde{a}^{\prime} \equiv a^{\prime}+\frac{\xi}{R}$ where $\xi>0$ and $a^{\prime}$ in terms of the constraint. Clearly $U\left(a^{\prime}+\frac{\xi}{R}, 0\right)$ and $U\left(\tilde{a}^{\prime}, 0\right)$ represent the same constraint, but $U\left(\tilde{a}^{\prime}, 0\right)$ and $U\left(a^{\prime}, 0\right)$ do not. In any case this is crucial only when $R=r$ is imposed, and not relevant in cases where we identified that the worker's constraint is slack.

42 As was previously discussed, frontloaded wages are the optimal contract also when $R=r$ (in fact in this case the upfront payments are even larger).
} 
the effect that the insurance contract has on the firm's profits and its sustainability constraints. ${ }^{4344}$ These two assumptions can be thought as additional frictions brought to the model, possibly not the only ones I could have utilized. However, the goal here is not to show that the model it self implies an equivalence between assets and severance payments, rather to show that the frontloaded profile is consistent with this interpretation.

To simplify I revisit the analytical model where unemployment is an absorbing state (see textSection 3.3). This assumption is innocuous for my results, it is employed here demonstrate the concepts more clearly. Moreover, it is convenient to utilize the Lagrangian approach to derive the results. This approach was described in a previous subsection.

Assume that every period the firm gives to the worker the option to purchase insurance whose price is $p$ and whose quantity (determined optimally by the worker) is $\xi_{t+1}$. This scheme pays $\xi_{t+1}$ in the event the firm and the worker separate. Moreover, as previously assume that severance payments can be enforced. Under these assumptions the firm's date zero implementability constraint can be written as:

$$
\sum_{t=0}^{\infty} \beta^{t}(1-\lambda)^{t}\left[y-w_{t}+\xi_{t+1} p-\beta \lambda \xi_{t+1}\right]=\Xi_{0}
$$

Moreover, the sequence of the firm's participation constraints is given by:

$$
\sum_{j=0}^{\infty} \beta^{j}(1-\lambda)^{j}\left[y-w_{t+j}+\xi_{t+j+1} p-\beta \lambda \xi_{t+j+1}\right] \geq 0 \quad \forall t \geq 0
$$

Note that the constraint in (34) equates the present value of profits for the firm to a number $\Xi_{0} \geq 0$. In the baseline model I had set $\Xi_{0}=0$, however, now I allow to have $\Xi_{0}>0$. The reason is that in the case where the worker purchases insurance, the firm saves earning a return $R>r$ and therefore creates wealth intertemporally. If we set the initial present value of profits to zero then the worker is entitled to a larger level of wages and consumption than under the baseline model. If I further assume that in the maximization problem the worker does not internalize the effect of severance payments on the firm's intertemporal constraints, ${ }^{45} \mathrm{I}$ can represent the maximization program as follows:

$$
\begin{gathered}
\mathcal{L}=\sum_{t=0}^{t=\infty}\left\{\beta^{t}(1-\lambda)^{t}\left[\log \left(-a_{t+1}+a_{t}-p \xi_{t+1}+w_{t}\right)+\beta \lambda U\left(a_{t+1}+\xi_{t+1}, 0\right)\right)\right. \\
\left.+\kappa_{t}\left(y-w_{t+j}+\tilde{\xi}_{t+j+1} p-\beta \lambda \tilde{\xi}_{t+j+1}\right)\right\} \\
-v \sum_{t=0}^{\infty} \beta^{t}(1-\lambda)^{t}\left[y-w_{t}+\tilde{\xi}_{t+1} p-\beta \lambda \tilde{\xi}_{t+1}\right]
\end{gathered}
$$

where $\kappa_{t}=\kappa_{t-1}+\phi_{t}$ and $\phi_{t}$ is the multiplier on the date $t$ participation constraint. Moreover, it holds that $\tilde{\xi}_{t+1}=\xi_{t+1}$ in equilibrium, but that the worker does not optimize with respect to $\tilde{\xi}_{t+1} \cdot{ }^{46}$

\footnotetext{
${ }^{43}$ The crucial issue is that even when $R=\frac{1}{\beta}>r$ the worker who promises to deliver zero profits to the firm, is happy to pay a higher premium for the insurance and then recover this premium in the form of higher wages. This is the rationale for the additional constraints imposed.

${ }^{44}$ In the version of the model I consider in the appendix firms may make positive profits up to the point where wages (in the baseline model) are frontloaded. The participation constraints are the same as previously.

${ }^{45}$ Without this the severance payment gives complete coverage against unemployment.

${ }^{46} \mathrm{I}$ have left out the participation constraint of the worker to simplify. This is not going to bind. Moreover, the borrowing constraint is also redundant. Further note that it is not important to impose that $\xi_{t+1} \geq 0$. Since profits will be negative if $\xi_{t+1}<0$ in a solution with constant severance payments this is ruled out from the firm's participation constraint.
} 
The first order conditions are:

$$
\begin{gathered}
-\frac{1}{c_{t}^{e}}+\beta \lambda U_{a_{t+1}+\xi_{t+1}}\left(a_{t+1}+\xi_{t+1}, 0\right)+\beta(1-\lambda) \frac{1}{c_{t+1}^{e}}=0 \\
\frac{1}{c_{t}^{e}}-\kappa_{t}+v=0 \\
-\frac{1}{c_{t}^{e}} p+\beta \lambda U_{a_{t+1}+\xi_{t+1}}\left(a_{t+1}+\xi_{t+1}, 0\right)=0
\end{gathered}
$$

From these equations we have i) Consumption is constant in the case where $\kappa_{t}=\kappa$ (constant) i.e. when the firm's participation constraint never binds. ii) the Euler equation holds as an equality and iii) the ratio of consumption in employment to unemployment is given by: $\frac{c_{t+1}^{e}}{c_{t+1}^{u}}=\frac{p}{\beta \lambda}$. Moreover, in the case where $p=\beta \lambda+(1-\beta)$ the drop in consumption is equivalent to the one derived in text.

Clearly these first order conditions are consistent with the wage profile derived analytically in text if we set $\xi=0$. In this case assets are utilized to hedge against unemployment, the worker accumulates in the first period $a_{1}=a_{0}+(y-\bar{w}) \frac{1}{1+\beta \lambda-\beta}$. Moreover, $\bar{w}=a_{0}(1-\beta)+\frac{1-\beta}{1-\beta+\beta \lambda}=c_{t}^{e}$ and $w_{0}>\bar{w}$. Therefore $a^{*} \equiv a_{1}=a_{0} \frac{\beta \lambda}{1-\beta+\beta \lambda}+y \frac{\beta \lambda}{(1-\beta+\beta \lambda)^{2}}$.

Let us now consider another solution to the first order conditions. Let the worker choose a positive level $\xi$ each period and let wages be constant throughout. Denote the constant wage level as $\overline{\bar{w}}$. To have the same insurance value this contract must set:

$$
a_{0}+\xi=a^{*} \rightarrow \xi=y \frac{\beta \lambda}{(1-\beta+\beta \lambda)^{2}}-\frac{a_{0}(1-\beta)}{(1-\beta+\beta \lambda)}
$$

Moreover, to have the same consumption level, it must be that $\overline{\bar{c}}_{t}^{e}=-p \xi+\overline{\bar{w}}=a_{0}(1-\beta)+\frac{1-\beta}{1-\beta+\beta \lambda}$, or:

$$
\overline{\bar{w}}=a_{0}(1-\beta)+\frac{1-\beta}{1-\beta+\beta \lambda}+(1-\beta+\beta \lambda) \xi=y
$$

Therefore, wages must equal productivity.

It is easy to check that this solution satisfies the first order conditions above. Moreover, it is straightforward to illustrate that the firm makes positive profits in equilibrium since it pays $y$ to the worker but gains in expectation $p \xi-\beta \lambda \xi=(1-\beta) \xi>0$ if $\xi>0$. Obviously profits are positive when assets $a_{0}$ are low enough so that the worker wishes to purchase insurance from the firm. If assets are high the firm's participation constraint binds, and the above derivations yield the decreasing consumption profile discussed in text.

These derivations show that frontloaded wages can be decentralized through severance payments, as was claimed in the text.

\section{B.8 Nash Bargaining}

The model presented in this paper assumed that the firm and the worker can commit to a given set of policies without ever renegotiating the optimal contract. Implicitly commitment is sustained by the threat of mutual reversion to autarky (see for example Ligon et al (2000)). This section illustrates the implications of allowing for renegotiation and bargaining between workers and firms in every period. I focus here on Nash bargaining following the bulk of the literature of search theoretic models. As is well known, under Nash bargaining the allocation is re-bargained in every period, this means that the firm and the worker cannot commit to any long term contract. The policy rules and payoffs will now not feature promised utility $J$ as a state variable, they will only be a function of the worker's asset endowment $a$. Therefore, the contract solved in this subsection is a Markov perfect equilibrium contract where wealth influences the payoffs. ${ }^{47}$

\footnotetext{
${ }^{47}$ A similar no-commitment contract is considered by Karaivanov and Martin (2015), albeit is a different setup without unemployment, and therefore no explicit reason for worker's to accumulate precautionary savings. Besides this difference, and the fact that my focus here is to highlight the properties of wages, the results in this subsection
} 
Let the equilibrium payoffs be $\Omega(a)$ and $\Phi(a)$ to the worker and the firm respectively. In order to uncover $\Omega(a)$ and $\Phi(a)$ I use a representation of the optimal contract as the (dual) problem of maximizing the firm's profit function (see section B.5 in the appendix). Moreover, I impose that the continuation policies are consistent with the equilibrium under Nash Bargaining. The program maybe written as follows:

$$
\Pi(W, a)=\max y-w+\frac{1-\lambda}{R} \Phi\left(a^{\prime}\right)
$$

subject to:

$$
\begin{gathered}
W \leq u\left(\frac{-a^{\prime}}{r}+w-\tau+a\right)+\beta\left((1-\lambda) \Omega\left(a^{\prime}\right)+\lambda U\left(a^{\prime}, 0\right)\right) \\
\Omega(a) \in \arg \max _{W}(W-U(a, 0))^{\eta}(\Pi(W, a))^{1-\eta} \\
\Phi(a)=\Pi(\Omega(a), a)
\end{gathered}
$$

(39) is the standard Nash Bargaining protocol. The firm and the worker share the surplus of the match through solving (39), where $\eta$ determines the share of the surplus that accrues to the worker. Equation (38) is the analogous object to the promise keeping constraint considered in text. It requires that at least a level of lifetime utility $W$ be delivered to the worker although in this case the continuation utility must be consistent with the equilibrium payoff $\Omega\left(a^{\prime}\right)$. Basically, (37) gives a solution to the firm's problem for any $W$. However, in equibrium the level of utility obtained by the worker must also satisfy (39). Therefore, the firm's profit is defined in equilibrium as $\Phi(a)=\Pi(\Omega(a), a)$. This is shown in equation (40)

This type of contract does not appear new in the literature. Krusell et al. (2010) and Nakajima (2012) construct models with search frictions in the labour market and incomplete insurance, assuming that rents are divided each period with a Nash protocol. However, their approach is different from mine; they assume from the outset that the Nash sharing rule gives a time invariant wage function $w(a)$ and subsequently solve the workers value function as:

$$
\Omega(a)=\max _{a^{\prime}} \log \left(-a^{\prime}+a r+w(a)\right)+\beta\left[\lambda U\left(a^{\prime}, 0\right)+(1-\lambda) \Omega\left(a^{\prime}\right)\right]
$$

Different from these authors, I treat allocations as part of a more general contracting problem. This does not impose object $w(a)$ from the outset and more importantly it allows to incorporate additional features to the model, such as considering separations endogenously determined by the worker's effort (see Wang and Williamson (2002) and Oikonomou (2010, Ch. 2)).

\section{B.8.1 Optimal allocation under Nash bargaining}

It is easy to show that optimal choices of $w$ and $a^{\prime}$ satisfy the following first order conditions:

$$
\begin{gathered}
\kappa_{t} u^{\prime}\left(c_{t}^{e}\right)=1 \\
\kappa_{t} \beta\left(\lambda U_{a_{t+1}}+(1-\lambda) \Omega_{a_{t+1}}\right)-\frac{1}{r}+\frac{1-\lambda}{R}\left(1-\kappa_{t+1} \Omega_{a_{t+1}}\right) \leq 0
\end{gathered}
$$

with strict equality if $a_{t+1}>0 . \quad \kappa_{t}$ represents the multiplier on the promise keeping constraint. The envelope condition is given by: $\Phi_{a_{t}}=1-\kappa_{t} \Omega_{a_{t}}$. These equations have the following interpretation: An increment is wealth in equation (42) has two distinct effects on the firm's profits: it lowers required wages to finance a given consumption stream, but also increases the level of promised utility that the firm must deliver to the worker (according to the derivative $\Omega_{a_{t+1}}$ ). The latter effect would tend to dominate the closer wealth is to the borrowing constraint, since it is precisely there that an increment in assets encounters the highest marginal utility gains. Rearranging (42) and making use

should be very similar to the results they obtain from their model. 
of the envelope conditions we get the following Euler condition for the model with Nash Bargaining:

$$
u^{\prime}\left(c_{t}^{e}\right) \geq \beta r\left(\lambda U_{a_{t+1}}+(1-\lambda) \Omega_{a_{t+1}}\right)+\frac{1-\lambda}{R} \frac{r}{\kappa_{t}} \Phi_{a_{t+1}}
$$

Equation (43) sets the marginal cost of saving an extra unit today, equal to the future marginal benefit, and an extra term that pertains to the shape of the profit function. Should $\Phi_{a_{t+1}}$ be less than zero, the marginal cost would be less than the marginal benefit and the agent would be savings constrained. The converse holds if $\Phi_{a_{t+1}}>0$.

\section{B.8.2 Consumption in unemployment}

It is more relevant to consider cases where the derivative $\Phi_{a_{t+1}}$ is less than zero. This is a common property of the numerical solutions to this model (see Krusell et al. (2010) and Oikonomou (2010 , Ch 1)). In this case we can show from the Nash rule that $\Omega_{a_{t+1}}-U_{a_{t+1}}<0$; in other words, the marginal benefit from an extra unit of wealth is higher for the unemployed than for the employed agent. Rearranging (42) we get:

$$
\kappa_{t+1} \Omega_{a_{t+1}}=1+\frac{\kappa_{t} R}{r(1-\lambda)} \beta r\left(\lambda U_{a_{t+1}}+(1-\lambda) \Omega_{a_{t+1}}-u^{\prime}\left(c_{t}^{e}\right)\right)<\kappa_{t+1} U_{a_{t+1}}
$$

Equation (44) gives the insurance value of the contract. Whenever $\Phi_{a_{t+1}}<0$ the term in the parenthesis is positive and consumption falls as the agent becomes unemployed.

\section{B.8.3 Wages}

The previous derivations cannot be further simplified to derive the behavior of wages analytically in the model. We can go so far as to claim that there is a wage rule $w(a)$ deriving from the contract considered in this subsection ${ }^{48}$, however, the exact form of $w(a)$ depends on the sign of the derivative $\Phi_{a_{t}}$ (and the analogous derivative of the worker's value function). As discussed previously, the properties of $w(a)$ have already been studied by Krusell et al (2002). The numerical solution to (37) delivers wage rules which display the properties highlighted in that paper (see Oikonomou (2010, Ch. 1)).

Of particular interest is the case where $\eta=1$. This is the only case where we can completely characterize the wage path. In particular we can show that the Nash bargaining contract is a flat wage contract; it sets wages equal to productivity each period. Assume the contrary: Let wages be frontloaded (i.e. $w_{0}>y$ initially) so that the worker receives a loan that finances wealth accumulation. Assume without loss of generality that the choice of assets is $a_{1}$. The firm's payoff is then $\Phi\left(a_{1}\right)$. It must be that $\Phi\left(a_{1}\right)=0$ since under $\eta=1$ firms break even under the rebargained allocation. However note that the equilibrium payoff satisfies $\Phi\left(a_{0}\right)=y-w_{0}+\frac{1-\lambda}{R} \Phi\left(a_{1}\right)<0$. This is a contradiction since the equilibrium payoff must also satisfy $\Phi\left(a_{0}\right)=0$.

Result 2. In an equilibrium under Nash Bargaining with $\eta=1$ the only incentive compatible allocation has wages equal to productivity each period (flat wage contract).

\footnotetext{
${ }^{48}$ This may be easily illustrated through taking a first order condition from 39 with respect to $W$ and making the appropriate substitutions.
} 


\section{B.9 Additional Tables and Figures}

Figure 4: Welfare Effects of Permanent Benefits

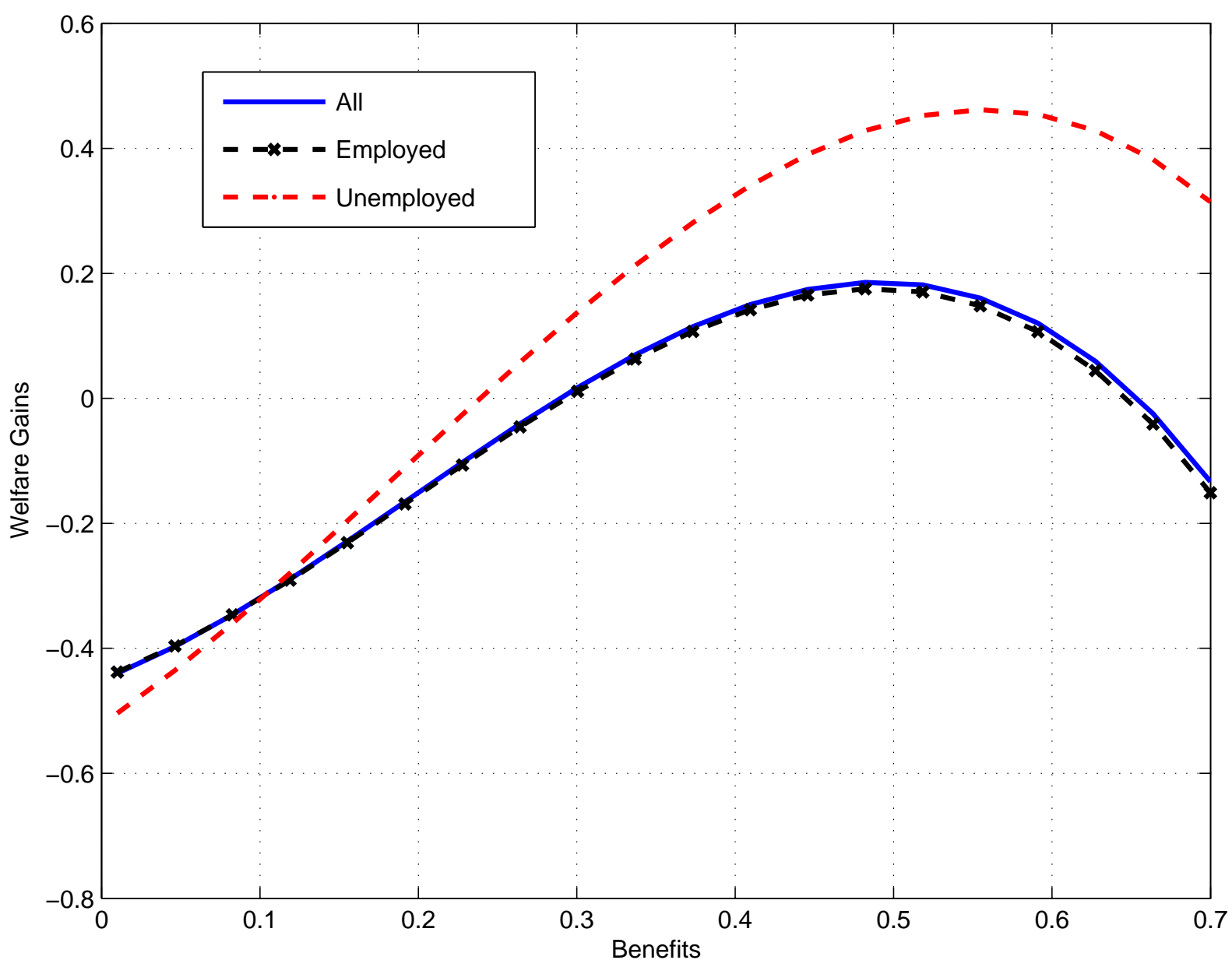

Notes: The figure shows the value of the compensating variation coefficient when we set $b_{j}=\bar{b}$ for all $j$. The $\mathrm{x}$ axis varies the level of 'permanent benefits'. The solid line shows the CE coefficient for all agents in the economy, the crossed line for employed individuals and the dashed line for unemployed agents. 
Table 4: Effects of UI Policies: $r-1=0.5 \%$

\begin{tabular}{lccccccccc} 
& Policy & $\tau$ & $u$ & $\% u_{\leq 1}$ & $\widetilde{\mu}$ & $\widetilde{\mu}_{e}$ & $\widetilde{\mu}_{u}$ & $w_{0}$ & Buffer \\
\hline \hline Benchmark & $b_{0}=b_{1}=0.5$ & $3.23 \%$ & $6.36 \%$ & $95.2 \%$ & 0 & 0 & 0 & 1.78 & 1.39 \\
\multirow{2}{*}{ Backloaded } & $b_{1}=0.5$ & $0.71 \%$ & $6.35 \%$ & $95.3 \%$ & $-0.22 \%$ & $-0.22 \%$ & $-0.22 \%$ & 2.24 & 2.06 \\
& $b_{1}=1$ & $1.77 \%$ & $6.88 \%$ & $93.5 \%$ & $-0.57 \%$ & $-0.57 \%$ & $-0.46 \%$ & 1.92 & 1.77 \\
Permanent & $b_{j}=0.5$ & $3.72 \%$ & $6.92 \%$ & $89.9 \%$ & $-0.20 \%$ & $-0.21 \%$ & $-0.04 \%$ & 1.51 & 0.76 \\
\multirow{2}{*}{ Frontloaded } & $b_{0}=1$ & $5.03 \%$ & $6.06 \%$ & $96.2 \%$ & $0.24 \%$ & $0.24 \%$ & $0.20 \%$ & 1.55 & 0.83 \\
\multirow{2}{*}{ Optimal } & $b_{0}=1 b_{j>0}=0.5$ & $6.24 \%$ & $6.94 \%$ & $89.9 \%$ & $-0.02 \%$ & $-0.04 \%$ & $0.20 \%$ & 1.04 & 0.10 \\
\hline \hline
\end{tabular}

Notes: The table shows the effects of various UI schemes. $b_{j}$ is the level of benefits given in period $j+1$ of an unemployment spell. Hence $j=0$ corresponds to benefits received during the first quarter, $j=1$ during the second and so on. $b^{*}$ denotes the optimal policy. As discussed in text we have $b_{0}^{*}=1.05 b_{j}^{*}=0, j>0$. For the remaining columns the level of unemployment benefits in $j$ is reported when $b_{j}>0$.

$\% u_{\leq 1}$ represents the fraction of unemployed individuals whose unemployment duration is less than or equal to 2 quarters. Following the notation in text, $\widetilde{\mu}$ is the percentage increment in consumption needed by individuals to keep the baseline UI policy.

Table 5: Average consumption in employment and unemployment: $r-1=0.5 \%$

\begin{tabular}{lcccccc} 
& Policy & $\% w_{0}>y$ & $\bar{c}^{e}$ & $\bar{c}_{o}^{u}$ & $\bar{c}_{1}^{u}$ & $\bar{c}_{\geq 2}^{u}$ \\
\hline \hline Benchmark & $b_{0}=b_{1}=0.5$ & $100 \%$ & 0.947 & 0.861 & 0.691 & 0.292 \\
\multirow{2}{*}{ Backloaded } & $b_{1}=0.5$ & $100 \%$ & 0.944 & 0.859 & 0.682 & 0.284 \\
& $b_{1}=1$ & $100 \%$ & 0.939 & 0.854 & 0.729 & 0.344 \\
Permanent & $b_{j}=0.5$ & $100 \%$ & 0.941 & 0.855 & 0.697 & 0.501 \\
Frontloaded & $b_{0}=1$ & $49 \%$ & 0.951 & 0.872 & 0.635 & 0.245 \\
\multirow{2}{*}{ Optimal } & $b_{0}=1 b_{j>0}=0.5$ & $37 \%$ & 0.940 & 0.878 & 0.734 & 0.509 \\
\hline \hline
\end{tabular}

Notes: The table shows the effects of various UI schemes on the average levels of consumption and the fraction of agents that are at the unconstrained optimum. $b_{j}$ is the level of benefits given in period $j+1$ of an unemployment spell. Hence $j=0$ corresponds to benefits received during the first quarter, $j=1$ during the second and so on. $b^{*}$ denotes the optimal policy. As discussed in text we have $b_{0}^{*}=1.05 b_{j}^{*}=0, j>0$. For the remaining columns the level of unemployment benefits in $j$ is reported when $b_{j}>0$.

$\bar{c}^{e}$ denotes average consumption of employed individuals. $\bar{c}_{j}^{u}$ denotes average consumption of unemployed agents where $j=0,1, \geq 2$ denotes the duration in unemployment. With $\geq 2$ I pool together all agents who have been unemployed for at least two quarters. 
Institut de Recherches Économiques et Sociales

Université catholique de Louvain

Place Montesquieu, 3

1348 Louvain-la-Neuve, Belgique 